\title{
Observation of Anisotropy in the Arrival Directions of Galactic Cosmic Rays at Multiple Angular Scales with IceCube
}

IceCube Collaboration: R. Abbasi ${ }^{1}$, Y. Abdou ${ }^{2}$, T. Abu-Zayyad ${ }^{3}$, J. Adams ${ }^{4}$, J. A. Aguilar ${ }^{1}$, M. Ahlers ${ }^{5}$, D. Altmann ${ }^{6}$, K. Andeen ${ }^{1}$, J. Auffenberg ${ }^{7}$, X. Bai ${ }^{8}$, M. Baker $^{1}$, S. W. Barwick ${ }^{9}$, R. Bay ${ }^{10}$, J. L. Bazo Alba ${ }^{11}$, K. Beattie ${ }^{12}$, J. J. Beatty ${ }^{13,14}$, S. Bechet ${ }^{15}$, J. K. Becker ${ }^{16}$, K.-H. Becker ${ }^{7}$, M. L. Benabderrahmane ${ }^{11}$, S. BenZvi ${ }^{1}$, J. Berdermann ${ }^{11}$, P. Berghaus ${ }^{8}$, D. Berley ${ }^{17}$, E. Bernardini ${ }^{11}$, D. Bertrand ${ }^{15}$, D. Z. Besson ${ }^{18}$, D. Bindig ${ }^{7}$, M. Bissok ${ }^{6}$, E. Blaufuss ${ }^{17}$, J. Blumenthal ${ }^{6}$, D. J. Boersma ${ }^{6}$, C. Bohm ${ }^{19}$, D. Bose ${ }^{20}$, S. Böser ${ }^{21}$, O. Botner ${ }^{22}$, A. M. Brown ${ }^{4}$, S. Buitink ${ }^{20}$, K. S. Caballero-Mora ${ }^{23}$, M. Carson ${ }^{2}$, D. Chirkin ${ }^{1}$, B. Christy ${ }^{17}$, J. Clem ${ }^{8}$, F. Clevermann ${ }^{24}$, S. Cohen ${ }^{25}$, C. Colnard ${ }^{26}$, D. F. Cowen ${ }^{23,27}$, M. V. D'Agostino ${ }^{10}$, M. Danninger ${ }^{19}$, J. Daughhetee ${ }^{28}$, J. C. Davis ${ }^{13}$, C. De Clercq $^{20}$, L. Demirörs ${ }^{25}$, T. Denger ${ }^{21}$, O. Depaepe ${ }^{20}$, F. Descamps ${ }^{2}$, P. Desiati ${ }^{1}$, G. de Vries-Uiterweerd ${ }^{2}$, T. DeYoung ${ }^{23}$,

J. C. Díaz-Vélez ${ }^{1}$, M. Dierckxsens ${ }^{15}$, J. Dreyer ${ }^{16}$, J. P. Dumm ${ }^{1}$, R. Ehrlich ${ }^{17}$, J. Eisch ${ }^{1}$, R. W. Ellsworth ${ }^{17}$, O. Engdegård ${ }^{22}$, S. Euler ${ }^{6}$, P. A. Evenson ${ }^{8}$, O. Fadiran ${ }^{29}$, A. R. Fazely ${ }^{30}$, A. Fedynitch ${ }^{16}$, J. Feintzeig ${ }^{1}$, T. Feusels ${ }^{2}$, K. Filimonov ${ }^{10}$, C. Finley ${ }^{19}$, T. Fischer-Wasels ${ }^{7}$, M. M. Foerster ${ }^{23}$, B. D. Fox $^{23}$, A. Franckowiak ${ }^{21}$, R. Franke ${ }^{11}$, T. K. Gaisser ${ }^{8}$, J. Gallagher ${ }^{31}$,

L. Gerhardt ${ }^{12,10}$, L. Gladstone ${ }^{1}$, T. Glüsenkamp ${ }^{6}$, A. Goldschmidt ${ }^{12}$, J. A. Goodman ${ }^{17}$,

D. Gora ${ }^{11}$, D. Grant ${ }^{32}$, T. Griesel ${ }^{33}$, A. Groß ${ }^{4,26}$, S. Grullon ${ }^{1}$, M. Gurtner ${ }^{7}$, C. Ha ${ }^{23}$,

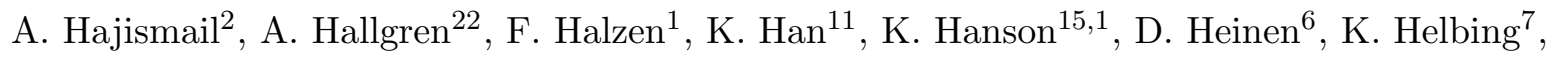
P. Herquet ${ }^{34}$, S. Hickford ${ }^{4}$, G. C. Hill ${ }^{1}$, K. D. Hoffman ${ }^{17}$, A. Homeier ${ }^{21}$, K. Hoshina ${ }^{1}$, D. Hubert ${ }^{20}$,

W. Huelsnitz ${ }^{17}$, J.-P. Hülß ${ }^{6}$, P. O. Hulth ${ }^{19}$, K. Hultqvist ${ }^{19}$, S. Hussain ${ }^{8}$, A. Ishihara ${ }^{35}$, J. Jacobsen ${ }^{1}$, G. S. Japaridze ${ }^{29}$, H. Johansson ${ }^{19}$, J. M. Joseph ${ }^{12}$, K.-H. Kampert ${ }^{7}$, A. Kappes ${ }^{36}$, T. Karg ${ }^{7}$, A. Karle ${ }^{1}$, P. Kenny ${ }^{18}$, J. Kiryluk ${ }^{12,10}$, F. Kislat ${ }^{11}$, S. R. Klein ${ }^{12,10}$, J.-H. Köhne ${ }^{24}$,

G. Kohnen ${ }^{34}$, H. Kolanoski ${ }^{36}$, L. Köpke ${ }^{33}$, S. Kopper ${ }^{7}$, D. J. Koskinen ${ }^{23}$, M. Kowalski²1,

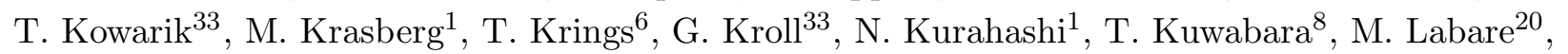

S. Lafebre ${ }^{23}$, K. Laihem ${ }^{6}$, H. Landsman ${ }^{1}$, M. J. Larson ${ }^{23}$, R. Lauer ${ }^{11}$, J. Lünemann ${ }^{33}$,

B. Madajczyk ${ }^{1}$, J. Madsen ${ }^{3}$, P. Majumdar ${ }^{11}$, A. Marotta ${ }^{15}$, R. Maruyama ${ }^{1}$, K. Mase $^{35}$,

H. S. Matis ${ }^{12}$, K. Meagher ${ }^{17}$, M. Merck ${ }^{1}$, P. Mészáros ${ }^{27,23}$, T. Meures ${ }^{15}$, E. Middell ${ }^{11}$, N. Milke ${ }^{24}$, J. Miller ${ }^{22}$, T. Montaruli ${ }^{1,37}$, R. Morse ${ }^{1}$, S. M. Movit ${ }^{27}$, R. Nahnhauer ${ }^{11}$, J. W. Nam ${ }^{9}$,

U. Naumann ${ }^{7}$, P. Nießen ${ }^{8}$, D. R. Nygren ${ }^{12}$, S. Odrowski ${ }^{26}$, A. Olivas ${ }^{17}$, M. Olivo ${ }^{16}$,

A. O’Murchadha ${ }^{1}$, M. Ono ${ }^{35}$, S. Panknin ${ }^{21}$, L. Paul ${ }^{6}$, C. Pérez de los Heros ${ }^{22}$, J. Petrovic ${ }^{15}$,

A. Piegsa ${ }^{33}$, D. Pieloth ${ }^{24}$, R. Porrata ${ }^{10}$, J. Posselt ${ }^{7}$, C. C. Price ${ }^{1}$, P. B. Price ${ }^{10}$,

G. T. Przybylski ${ }^{12}$, K. Rawlins ${ }^{38}$, P. Redl ${ }^{17}$, E. Resconi ${ }^{26}$, W. Rhode ${ }^{24}$, M. Ribordy ${ }^{25}$, A. Rizzo $^{20}$, J. P. Rodrigues ${ }^{1}$, P. Roth ${ }^{17}$, F. Rothmaier ${ }^{33}$, C. Rott ${ }^{13}$, T. Ruhe ${ }^{24}$, D. Rutledge ${ }^{23}$, B. Ruzybayev ${ }^{8}$, D. Ryckbosch ${ }^{2}$, H.-G. Sander ${ }^{33}$, M. Santander ${ }^{1}$, S. Sarkar ${ }^{5}$, K. Schatto ${ }^{33}$, T. Schmidt ${ }^{17}$,

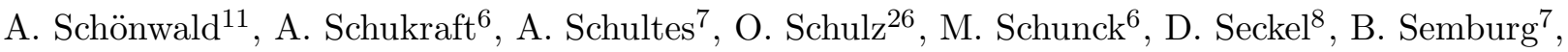
S. H. Seo ${ }^{19}$, Y. Sestayo ${ }^{26}$, S. Seunarine ${ }^{39}$, A. Silvestri ${ }^{9}$, A. Slipak ${ }^{23}$, G. M. Spiczak ${ }^{3}$, C. Spiering ${ }^{11}$, M. Stamatikos ${ }^{13,40}$, T. Stanev ${ }^{8}$, G. Stephens ${ }^{23}$, T. Stezelberger ${ }^{12}$, R. G. Stokstad ${ }^{12}$, A. Stössl ${ }^{11}$,

S. Stoyanov ${ }^{8}$, E. A. Strahler ${ }^{20}$, T. Straszheim ${ }^{17}$, M. Stür ${ }^{21}$, G. W. Sullivan ${ }^{17}$, Q. Swillens ${ }^{15}$,

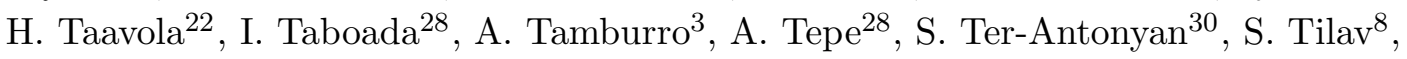


P. A. Toale ${ }^{41}$, S. Toscano ${ }^{1}$, D. Tosi ${ }^{11}$, D. Turčan ${ }^{17}$, N. van Eijndhoven ${ }^{20}$, J. Vandenbroucke ${ }^{10}$, A. Van Overloop ${ }^{2}$, J. van Santen ${ }^{1}$, M. Vehring ${ }^{6}$, M. Voge ${ }^{21}$, C. Walck ${ }^{19}$, T. Waldenmaier ${ }^{36}$, M. Wallraff ${ }^{6}$, M. Walter ${ }^{11}$, Ch. Weaver ${ }^{1}$, C. Wendt ${ }^{1}$, S. Westerhoff ${ }^{1}$, N. Whitehorn ${ }^{1}$, K. Wiebe ${ }^{33}$, C. H. Wiebusch ${ }^{6}$, D. R. Williams ${ }^{41}$, R. Wischnewski ${ }^{11}$, H. Wissing ${ }^{17}$, M. Wolf ${ }^{26}$, T. R. Wood $^{32}$, K. Woschnagg ${ }^{10}$, C. Xu ${ }^{8}$, X. W. Xu ${ }^{30}$, G. Yodh $^{9}$, S. Yoshida ${ }^{35}$, P. Zarzhitsky ${ }^{41}$, and M. Zoll ${ }^{19}$ 


\footnotetext{
${ }^{1}$ Dept. of Physics, University of Wisconsin, Madison, WI 53706, USA

${ }^{2}$ Dept. of Physics and Astronomy, University of Gent, B-9000 Gent, Belgium

${ }^{3}$ Dept. of Physics, University of Wisconsin, River Falls, WI 54022, USA

${ }^{4}$ Dept. of Physics and Astronomy, University of Canterbury, Private Bag 4800, Christchurch, New Zealand

${ }^{5}$ Dept. of Physics, University of Oxford, 1 Keble Road, Oxford OX1 3NP, UK

${ }^{6}$ III. Physikalisches Institut, RWTH Aachen University, D-52056 Aachen, Germany

${ }^{7}$ Dept. of Physics, University of Wuppertal, D-42119 Wuppertal, Germany

${ }^{8}$ Bartol Research Institute and Department of Physics and Astronomy, University of Delaware, Newark, DE 19716, USA

${ }^{9}$ Dept. of Physics and Astronomy, University of California, Irvine, CA 92697, USA

${ }^{10}$ Dept. of Physics, University of California, Berkeley, CA 94720, USA

${ }^{11}$ DESY, D-15735 Zeuthen, Germany

${ }^{12}$ Lawrence Berkeley National Laboratory, Berkeley, CA 94720, USA

${ }^{13}$ Dept. of Physics and Center for Cosmology and Astro-Particle Physics, Ohio State University, Columbus, OH 43210, USA

${ }^{14}$ Dept. of Astronomy, Ohio State University, Columbus, OH 43210, USA

${ }^{15}$ Université Libre de Bruxelles, Science Faculty CP230, B-1050 Brussels, Belgium

${ }^{16}$ Fakultät für Physik \& Astronomie, Ruhr-Universität Bochum, D-44780 Bochum, Germany

${ }^{17}$ Dept. of Physics, University of Maryland, College Park, MD 20742, USA

${ }^{18}$ Dept. of Physics and Astronomy, University of Kansas, Lawrence, KS 66045, USA

${ }^{19}$ Oskar Klein Centre and Dept. of Physics, Stockholm University, SE-10691 Stockholm, Sweden

${ }^{20}$ Vrije Universiteit Brussel, Dienst ELEM, B-1050 Brussels, Belgium

${ }^{21}$ Physikalisches Institut, Universität Bonn, Nussallee 12, D-53115 Bonn, Germany

${ }^{22}$ Dept. of Physics and Astronomy, Uppsala University, Box 516, S-75120 Uppsala, Sweden

${ }^{23}$ Dept. of Physics, Pennsylvania State University, University Park, PA 16802, USA

${ }^{24}$ Dept. of Physics, TU Dortmund University, D-44221 Dortmund, Germany

${ }^{25}$ Laboratory for High Energy Physics, École Polytechnique Fédérale, CH-1015 Lausanne, Switzerland

${ }^{26}$ Max-Planck-Institut für Kernphysik, D-69177 Heidelberg, Germany

${ }^{27}$ Dept. of Astronomy and Astrophysics, Pennsylvania State University, University Park, PA 16802, USA

${ }^{28}$ School of Physics and Center for Relativistic Astrophysics, Georgia Institute of Technology, Atlanta, GA 30332, USA

${ }^{29}$ CTSPS, Clark-Atlanta University, Atlanta, GA 30314, USA

${ }^{30}$ Dept. of Physics, Southern University, Baton Rouge, LA 70813, USA

${ }^{31}$ Dept. of Astronomy, University of Wisconsin, Madison, WI 53706, USA
} 


\begin{abstract}
Between May 2009 and May 2010, the IceCube neutrino detector at the South Pole recorded 32 billion muons generated in air showers produced by cosmic rays with a median energy of $20 \mathrm{TeV}$. With a data set of this size, it is possible to probe the southern sky for per-mille anisotropy on all angular scales in the arrival direction distribution of cosmic rays. Applying a power spectrum analysis to the relative intensity map of the cosmic ray flux in the southern hemisphere, we show that the arrival direction distribution is not isotropic, but shows significant structure on several angular scales. In addition to previously reported large-scale structure in the form of a strong dipole and quadrupole, the data show small-scale structure on scales between $15^{\circ}$ and $30^{\circ}$. The skymap exhibits several localized regions of significant excess and deficit in cosmic ray intensity. The relative intensity of the smaller-scale structures is about a factor of 5 weaker than that of the dipole and quadrupole structure. The most significant structure, an excess localized at (right ascension $\alpha=122.4^{\circ}$ and declination $\delta=-47.4^{\circ}$ ), extends over at least $20^{\circ}$ in right ascension and has a post-trials significance of $5.3 \sigma$. The origin of this anisotropy is still unknown.
\end{abstract}

Subject headings: astroparticle physics — cosmic rays

\title{
1. Introduction
}

The IceCube detector, deployed $1450 \mathrm{~m}$ below the surface of the South Polar ice sheet, is designed to detect upward-going neutrinos from astrophysical sources. However, it is also sensitive to downward-going muons produced in cosmic ray air showers. To penetrate the ice and trigger the detector, the muons must possess an energy of at least several hundred GeV, which means they

\footnotetext{
${ }^{32}$ Dept. of Physics, University of Alberta, Edmonton, Alberta, Canada T6G 2G7

${ }^{33}$ Institute of Physics, University of Mainz, Staudinger Weg 7, D-55099 Mainz, Germany

${ }^{34}$ Université de Mons, 7000 Mons, Belgium

${ }^{35}$ Dept. of Physics, Chiba University, Chiba 263-8522, Japan

${ }^{36}$ Institut für Physik, Humboldt-Universität zu Berlin, D-12489 Berlin, Germany

${ }^{37}$ also Università di Bari and Sezione INFN, Dipartimento di Fisica, I-70126, Bari, Italy

${ }^{38}$ Dept. of Physics and Astronomy, University of Alaska Anchorage, 3211 Providence Dr., Anchorage, AK 99508 , USA

${ }^{39}$ Dept. of Physics, University of the West Indies, Cave Hill Campus, Bridgetown BB11000, Barbados

${ }^{40}$ NASA Goddard Space Flight Center, Greenbelt, MD 20771, USA

${ }^{41}$ Dept. of Physics and Astronomy, University of Alabama, Tuscaloosa, AL 35487, USA
} 
are produced by primary cosmic rays with energies in excess of several TeV. Simulations show that the detected direction of an air shower muon is typically within $0.2^{\circ}$ of the direction of the primary particle, so the arrival direction distribution of muons recorded in the detector is also a map of the cosmic ray arrival directions between about $1 \mathrm{TeV}$ and several $100 \mathrm{TeV}$. IceCube is currently the only instrument that can produce such a skymap of cosmic ray arrival directions in the southern sky. It records several $10^{10}$ cosmic ray events per year, which makes it possible to study anisotropy in the arrival direction distribution at the $10^{-4}$ level and below.

It is believed that charged cosmic rays at $\mathrm{TeV}$ energies are accelerated in supernova remnants in the Galaxy. It is also expected that interactions of cosmic rays with Galactic magnetic fields should completely scramble their arrival directions. For example, the Larmor radius of a proton with $10 \mathrm{TeV}$ energy in a $\mu \mathrm{G}$ magnetic field is approximately $0.01 \mathrm{pc}$, orders of magnitude less than the distance to any potential accelerator. Nevertheless, multiple observations of anisotropy in the arrival direction distribution of cosmic rays have been reported on large and small angular scales, mostly from detectors in the northern hemisphere. These deviations from isotropy in the cosmic ray flux between several $\mathrm{TeV}$ and several hundred $\mathrm{TeV}$ are at the part-per-mille level, according to data from the Tibet AS $\gamma$ array (Amenomori et al. 2005, 2006), the Super-Kamiokande Detector (Guillian et al. 2007), the Milagro Gamma Ray Observatory (Abdo et al. 2008, 2009), ARGO-YBJ (Vernetto et al. 2009), and EAS-TOP (Aglietta et al. 2009). Recently, a study of muons observed with the IceCube detector has revealed a large-scale anisotropy in the southern sky that is similar to that detected in the north (Abbasi et al. 2010b).

In this paper, we present the results of a search for cosmic ray anisotropy on all scales in the southern sky with data recorded between May 2009 and May 2010 with the IceCube detector in its 59-string configuration. An angular power spectrum analysis reveals that the cosmic ray skymap as observed by IceCube is dominated by a strong dipole and quadrupole moment, but it also exhibits significant structure on scales down to about $15^{\circ}$. This small-scale structure is about a factor 5 weaker in relative intensity than the dipole and quadrupole and becomes visible when these large-scale structures are subtracted from the data. A comprehensive search for deviations of the cosmic ray flux from isotropy on all angular scales reveals several localized regions of cosmic ray excess and deficit, with a relative intensity of the order of $10^{-4}$. The most significant structure is located at right ascension $\alpha=122.4^{\circ}$ and declination $\delta=-47.4^{\circ}$ and has a significance of $5.3 \sigma$ after correcting for trials. A comparison with data taken with fewer strings in the two years prior to this period confirms that these structures are a persistent feature of the southern sky.

The paper is organized as follows. In this section, we give a short summary of previous observations, almost exclusively in the northern hemisphere, of anisotropy in the cosmic ray arrival skymap at $\mathrm{TeV}$ energies. After the description of the IceCube detector and the data set used for this analysis (Section 2), the analysis techniques and results are presented in Section 3. In Section4, we show the outcome of several systematic checks of the analysis. The results are summarized and compared to Milagro results in the northern hemisphere in Section 5 . 


\subsection{Past Observations of Large- and Small-Scale Anisotropy}

The presence of a large-scale anisotropy in the distribution of charged cosmic rays can be caused by several effects. For example, configurations of the heliospheric magnetic field and other fields in the neighborhood of the solar system may be responsible. In this case, it is expected that the strength of the anisotropy should weaken with energy due to the increasing magnetic rigidity of the primary particles. The present data cannot unambiguously support or refute this hypothesis. Measurements from the Tibet AS $\gamma$ experiment indicate that the anisotropy disappears above a few hundred TeV (Amenomori et al. 2006), but a recent analysis of EAS-TOP data appears to show an increase in the amplitude of the anisotropy above $400 \mathrm{TeV}$ (Aglietta et al. 2009).

Existing data sets have also been searched for a time-dependent modulation of the anisotropy, which could be due to solar activity perhaps correlated with the eleven-year solar cycle. Results are inconclusive at this point. Whereas the Milagro data exhibit an increase in the mean depth of a large deficit region in the field of view over time (Abdo et al. 2009), no variation of the anisotropy with the solar cycle has been observed in Tibet AS $\gamma$ data (Amenomori et al. 2010). If these results are confirmed with more data recorded over longer time periods, different structures might show a different long-term behavior.

A large-scale anisotropy can also be caused by any relative motion of the Earth through the rest frame of the cosmic rays. The intensity of the cosmic ray flux should be enhanced in the direction of motion and reduced in the opposite direction, causing a dipole anisotropy in the coordinate frame where the direction of motion is fixed. However, the Earth's motion through space is complex and a superposition of several components, and the rest frame of the cosmic ray plasma is not known. If we assume the cosmic rays are at rest with respect to the Galactic Center, then a dipole of amplitude $0.35 \%$ should be observed due to the solar orbit about the Galactic Center. Such a dipole anisotropy, which would be inclined at about $45^{\circ}$ with respect to the celestial equator, was first proposed by Compton \& Getting (1935). Although the effect is strong enough to be measured by modern detectors, it has not been observed. This null result likely indicates that galactic cosmic rays co-rotate with the local Galactic magnetic field (Amenomori et al. 2006).

The motion of the Earth around the Sun also causes a dipole in the arrival directions of cosmic rays. The dipole is aligned with the ecliptic plane, and its strength is expected to be of order $10^{-4}$. This solar dipole effect has been observed by the Tibet AS $\gamma$ experiment (Amenomori et al. 2004) and Milagro (Abdo et al. 2009) and provides a sensitivity test for all methods looking for large-scale anisotropy in equatorial coordinates.

In addition to the large-scale anisotropy, data from several experiments in the northern hemisphere indicate the presence of small-scale structures with scales of order $10^{\circ}$. Using seven years of data, the Milagro collaboration published the detection of two regions of enhanced flux with

amplitude $10^{-4}$ and a median energy of $1 \mathrm{TeV}$ with significance $>10 \sigma$ (Abdo et al. 2008). The same excess regions also appear on skymaps produced by ARGO-YBJ (Vernetto et al. 2009). 
Small-scale structures in the arrival direction distribution may indicate nearby sources of cosmic rays, although the small Larmor radius at $\mathrm{TeV}$ energies makes it impossible for these particles to point back to their sources unless some unconventional propagation mechanism is assumed (Malkov et al. 2010). Diffusion from nearby supernova remnants, magnetic funneling (Drury \& Aharonian 2008), and cosmic ray acceleration from magnetic reconnection in the solar magnetotail (Lazarian \& Desiati 2010) have all been suggested as possible causes for the small-scale structure in the northern hemisphere.

\subsection{Analysis Techniques}

While the presence of large-scale structure in the southern sky has already been established using IceCube data (Abbasi et al. 2010b), there has not been a search of the southern sky for correlations on smaller angular scales. In this paper, we present a comprehensive study of the cosmic ray arrival directions in IceCube which includes, but is not limited to, the search for smallscale structures.

Large and small-scale structure have traditionally been analyzed with very different methods. The presence of a large-scale anisotropy is usually established by fitting the exposurecorrected arrival direction distribution in right ascension to the first few elements of a harmonic

series (Amenomori et al. 2006). While essentially a one-dimensional method, the procedure can be applied to the right ascension distribution in several declination bands to probe the strength of dipole and quadrupole moments as a function of declination (Abdo et al. 2009). To search for small-scale structure, the estimation for an isotropic sky is compared to the actual arrival direction distribution to find significant deviations from isotropy (Abdo et al. 2008; Vernetto et al. 2009).

Since both the large and small-scale structure in the cosmic ray data are currently unexplained, it is not obvious whether a "clean" separation between large and small scales is the right approach. The anisotropy in the arrival direction distribution might be a superposition of several effects, with the small-scale structure being caused by a different mechanism than the large-scale structure, or it might be the result of a single mechanism producing a complex skymap with structure on all scales.

The analysis presented in this paper makes use of a number of complementary methods to study the arrival direction distribution without prior separation into searches for large and smallscale structure. The basis of this study is the angular power spectrum of the arrival direction distribution. A power spectrum analysis decomposes the skymap into spherical harmonics and provides information on the angular scale of the anisotropy in the map. The power spectrum indicates which multipole moments $\ell=(0,1,2, \ldots)$ in the spherical harmonic expansion contribute significantly to the observed arrival direction distribution. To produce a skymap of the contribution of the $\ell \geq 3$ multipoles, the strong contributions from the dipole $(\ell=1)$ and quadrupole $(\ell=2)$ have to be subtracted first. The residual map can then be studied for structure on angular scales 
corresponding to $\ell \geq 3$. This is the first search for structure at these scales in the arrival direction distribution of $\mathrm{TeV}$ cosmic rays in the southern sky.

\section{The IceCube Detector}

IceCube is a $\mathrm{km}^{3}$-size neutrino detector frozen into the glacial ice sheet at the geographic South Pole. The ice serves as the detector medium. High-energy neutrinos are detected by observing the Cherenkov radiation from charged particles produced by neutrino interactions in the ice or in the bedrock below the detector.

The Cherenkov light is detected by an array of Digital Optical Modules (DOMs) embedded in the ice. Each DOM is a pressure-resistant glass sphere that contains a $25 \mathrm{~cm}$ photomultiplier tube (PMT) (Abbasi et al. 2010a) and electronics which digitize, timestamp, and transmit signals to the data acquisition system (Abbasi et al. 2009b). The IceCube array contains 5160 DOMs deployed at depths between $1450 \mathrm{~m}$ and $2450 \mathrm{~m}$ below the surface of the ice sheet. The DOMs are attached to 86 vertical cables, or strings, which are used for deployment and to transmit data to the surface. The horizontal distance between strings in the standard detector geometry is about $125 \mathrm{~m}$, while the typical vertical spacing between consecutive DOMs in each string is about $17 \mathrm{~m}$. Six strings are arranged into a more compact configuration, with smaller spacing between DOMs, at the bottom of the detector, forming DeepCore, designed to extend the energy reach of IceCube to lower neutrino energies. On the ice surface sits IceTop, an array of detectors dedicated to the study of the energy spectrum and composition of cosmic rays with energies between $500 \mathrm{TeV}$ and $1 \mathrm{EeV}$, several orders of magnitude larger in energy than the cosmic rays studied in this analysis. All data used in this work comes from the IceCube in-ice detector only.

Construction of IceCube has recently been completed with all 86 strings deployed. The detector has been operating in various configurations since 2005 (Achterberg et al. 2006). Between 2007 and 2008, it operated with 22 strings deployed (IC22), between 2008 and 2009 with 40 strings (IC40), and between 2009 and 2010 with 59 strings (IC59).

IceCube is sensitive to all neutrino flavors. Muon neutrinos, identified by the "track-like" signature of the muon produced in a charged-current interaction, form the dominant detection channel. Muons produced by astrophysical neutrinos are detected against an overwhelming background of muons produced in cosmic ray air showers in the atmosphere above the detector. IceCube searches are most sensitive to neutrino sources in the northern hemisphere, where the Earth can be used as a filter against atmospheric muons (Abbasi et al. 2009a).

While atmospheric muons are a background for neutrino astrophysics, they are a valuable tool in the analysis of the cosmic rays that produce them. The downgoing muons preserve the direction of the cosmic ray air shower, and thus the cosmic ray primary, and can be used to study the arrival direction distribution of cosmic rays at energies above roughly $10 \mathrm{TeV}$. 


\subsection{DST Data Set}

The trigger rate of downgoing muons is about $0.5 \mathrm{kHz}$ in IC22, $1.1 \mathrm{kHz}$ in IC40, and $1.7 \mathrm{kHz}$ in IC59. This rate is of order $10^{6}$ times the neutrino rate, and too large to allow for storage of the raw data. Instead, downgoing muon events are stored in a separate Data Storage and Transfer (DST) format suitable for recording high-rate data at the South Pole. The DST format is used to store the results of an online reconstruction performed on all events that trigger the IceCube detector. Most of the data are downward-going muons produced by cosmic ray air showers. Because of the high trigger rate, the DST filter stream is used to save a very limited set of information for every event. Basic event parameters such as energy estimators are stored, while digitized waveforms are only transmitted for a limited subset of events. The data are encoded in a compressed format that allows for the transfer of about 3 GB per day via the South Pole Archival and Data Exchange (SPADE) satellite communication system.

The main trigger used for physics analysis in IceCube is a simple majority trigger which requires coincidence of 8 or more DOMs hit in the deep ice within a $5 \mu$ s window. In order to pass the trigger condition, those hits have to be in coincidence with at least one other hit in the nearest or next-to-nearest neighboring DOM within $\pm 1 \mu s$ (local coincidence hits). Triggered events are reconstructed using two fast on-line algorithms (Ahrens et al. 2004). The first reconstruction is a line-fit algorithm based on an analytic $\chi^{2}$ minimization. It produces an initial event track from the position and timing of the hits and the total charge, but it does not account for the geometry of the Cherenkov cone and the scattering and absorption of photons in the ice. The second algorithm is a maximum likelihood-based muon track reconstruction, seeded with the linefit estimate of the arrival direction. The likelihood reconstruction is more accurate, but also more computationally expensive, so it is applied only when at least ten optical sensors are triggered by the event. The analysis presented in this work uses only events reconstructed with the maximum likelihood algorithm.

In addition to particle arrival directions, the DST data also contain the number of DOMs and hits participating in the event, as well as the total number of triggered strings, and the position of the center of gravity of the event. The number of DOMs in the event can be used as a measure of the energy of the primary cosmic ray. Above $1 \mathrm{TeV}$, the energy resolution is of order of 0.5 in $\Delta(\log (E))$, where $E$ is the energy of the primary cosmic ray. Most of the uncertainty originates in the physics of the air shower. In this energy range, we are dominated by air showers containing muons with energies near the threshold necessary to reach the deep ice. Fluctuations in the generation of these muons are the main contribution to the uncertainty in the determination of the energy of the primary cosmic ray. 

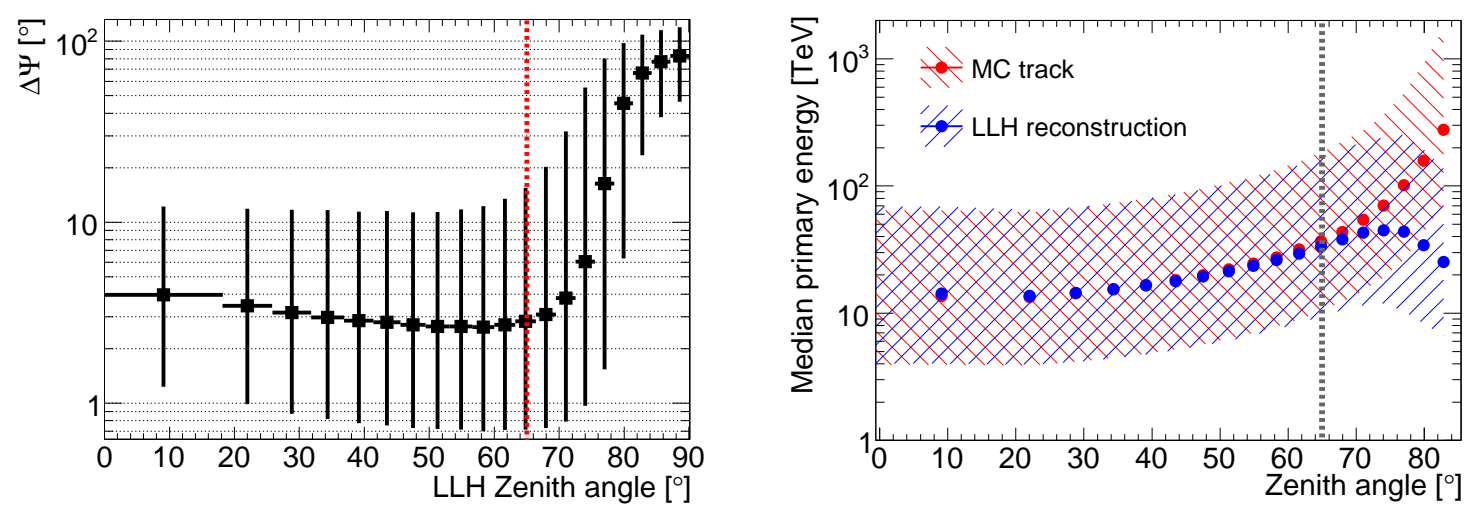

Fig. 1.- Median angular resolution (left) and median energy (right) as a function of zenith angle for simulated cosmic ray events. The error bars on the left plot and the hatched regions on the right one correspond to a $68 \%$ containing interval. The median primary energy is shown both as a function of the true zenith angle (MC track) and the reconstructed zenith angle (LLH reconstruction), while the median angular resolution (left) is shown as a function of the reconstructed zenith angle only. The dotted vertical line at $\theta=65^{\circ}$ indicates the cut in zenith angle performed in this work.

\subsection{Data Quality Cuts, Median Energy and Angular Resolution}

The analysis presented in this paper uses the DST data collected during IC59 operations between 2009 May 20 and 2010 May 30. The data set contains approximately $3.4 \times 10^{10}$ muon events detected with an integrated livetime of 334.5 days. A cut in zenith angle to remove misreconstructed tracks near the horizon (see below) reduces the final data set to $3.2 \times 10^{10}$ events.

Simulated air showers are used to evaluate the median angular resolution of the likelihood reconstruction and the median energy of the downgoing muon DST data set. The simulated data are created using the standard air shower Monte Carlo program CORSIKA 1 (Heck et al. 1998). The cosmic ray spectrum and composition are simulated using the polygonato model of Hörandel (2003), and the air showers are generated with the SIBYLL model of high-energy hadronic interactions (Ahn et al. 2009).

The simulations show that, for zenith angles smaller than $65^{\circ}$, the median angular resolution is $3^{\circ}$. This is not to be confused with the angular resolution of IceCube for neutrino-induced tracks (better than $1^{\circ}$ ), where more sophisticated reconstruction algorithms and more stringent quality cuts are applied. The resolution depends on the zenith angle of the muon. Fig. 1 (left) shows the median angular resolution as a function of zenith angle. The resolution improves from $4^{\circ}$ at small zenith angles to about $2.5^{\circ}$ near $60^{\circ}$. The larger space angle error at small zenith angles is caused

\footnotetext{
${ }^{1}$ COsmic Ray SImulations for KAscade: http://www-ik.fzk.de/corsika/
} 
by the detector geometry, which makes it difficult to reconstruct the azimuth angle for near-vertical showers. Consequently, with the azimuth angle being essentially unknown, the angular error can be large. For zenith angles greater than $65^{\circ}$, the angular resolution degrades markedly. The reason is that more and more events with apparent zenith angle greater than $65^{\circ}$ are misreconstructed tracks of smaller zenith angle and lower energy. The energy threshold for muon triggers increases rapidly with slant depth in the atmosphere and ice, and the statistics at large zenith angle become quite poor. We restrict our analysis to events with zenith angles smaller than $65^{\circ}$. Within this range, the angular resolution is roughly constant and much smaller than the angular size of arrival direction structure we are trying to study.

Using simulated data, we estimate that the overall median energy of the primary cosmic rays that trigger the IceCube detector is $20 \mathrm{TeV}$. Simulations show that at this energy the detector is more sensitive to protons than to heavy nuclei like iron. The median energy increases monotonically with the true zenith angle of the primary particle (Fig. 1 (right)) due to the attenuation of low-energy muons with increasing slant depth of the atmosphere and ice. The median energy also increases as a function of reconstructed zenith angle. Near the horizon, the large fraction of misreconstructed events causes the median energy to fall.

\section{Analysis}

The arrival direction distribution of cosmic rays observed by detectors like IceCube is not isotropic. Nonuniform exposure to different parts of the sky, gaps in the uptime, and other detectorrelated effects will cause a spurious anisotropy in the measured arrival direction distribution even if the true cosmic ray flux is isotropic. Consequently, in any search for anisotropy in the cosmic ray flux, these detector-related effects need to be accounted for. The first step in this search is therefore the creation of a "reference map" to which the actual data map is compared. The reference map essentially shows what the skymap would look like if the cosmic ray flux was isotropic. It is not in itself isotropic, because it includes the detector effects mentioned above. The reference map must be subtracted from the real skymap in order to find regions where the actual cosmic ray flux deviates from the isotropic expectation.

In this section, we first describe the construction of the reference map for the subsequent analysis. The reference map is then compared to the actual data map, and a map of the relative cosmic ray intensity is produced. We then perform several analyses to search for the presence of significant anisotropy in the relative intensity map.

\subsection{Calculation of the Reference Level}

For the construction of a reference map that represents the detector response to an isotropic sky, it is necessary to determine the exposure of the detector as a function of time and integrate it 
over the livetime. We use the method of Alexandreas et al. (1993) to calculate the exposure from real data. This technique is commonly used in $\gamma$-ray astronomy to search for an excess of events above the exposure-weighted isotropic reference level.

The method works as follows. The sky is binned into a fine grid in equatorial coordinates (right ascension $\alpha$, declination $\delta$ ). Two sky maps are then produced. The data map $N(\alpha, \delta)$ stores the arrival directions of all detected events. For each detected event that is stored in the data map, 20 "fake" events are generated by keeping the local zenith and azimuth angles $(\theta, \phi)$ fixed and calculating new values for right ascension using times randomly selected from within a pre-defined time window $\Delta t$ bracketing the time of the event being considered. These fake events are stored in the reference map with a weight of $1 / 20$. Using 20 fake events per real event, the statistical error on the reference level can be kept small.

Created in this way, the events in the reference map have the same local arrival direction distribution as the real events. Furthermore, this "time scrambling" method naturally compensates for variations in the event rate, including the presence of gaps in the detector uptime. The buffer length $\Delta t$ needs to be chosen such that the detector conditions remain stable within this period. Due to its unique location at the South Pole, the angular acceptance of IceCube is stable over long periods. The longest $\Delta t$ used in this analysis is one day, and the detector stability over this time period has been verified by $\chi^{2}$-tests comparing the arrival direction distributions at various times inside the window. The IceCube detector is, in fact, stable over periods longer than 24 hours.

Deviations from isotropy are known to bias estimates of the reference level produced by this method. In the vicinity of a strong excess, the method can create artificial deficits, as the events from the excess region are included in the estimation of the reference level. Similarly, there can be artificial excesses near strong deficits. In searches for point sources, the effect is usually negligible, but it can become significant in the presence of extended regions of strong excess or deficit flux.

Since the Earth rotates by $15^{\circ}$ every hour, the right ascension range of the scrambled data is $15^{\circ} /$ hour $\times \Delta t$, so any structure in the data map that is larger than $15^{\circ} /$ hour $\times \Delta t$ will also appear in the reference map and therefore be suppressed in the relative intensity map $\Delta N /\langle N\rangle$. For example, $\Delta t=2 \mathrm{hr}$ will suppress structures larger than $30^{\circ}$ in the relative intensity map. To be sensitive to large-scale structure such as a dipole, a time window of 24 hours (or higher) must be used.

\subsection{Relative Intensity and Significance Maps}

Once the data and reference maps are calculated, deviations from isotropy can be analyzed by calculating the relative intensity

$$
\frac{\Delta N_{i}}{\langle N\rangle_{i}}=\frac{N_{i}(\alpha, \delta)-\left\langle N_{i}(\alpha, \delta)\right\rangle}{\left\langle N_{i}(\alpha, \delta)\right\rangle}
$$



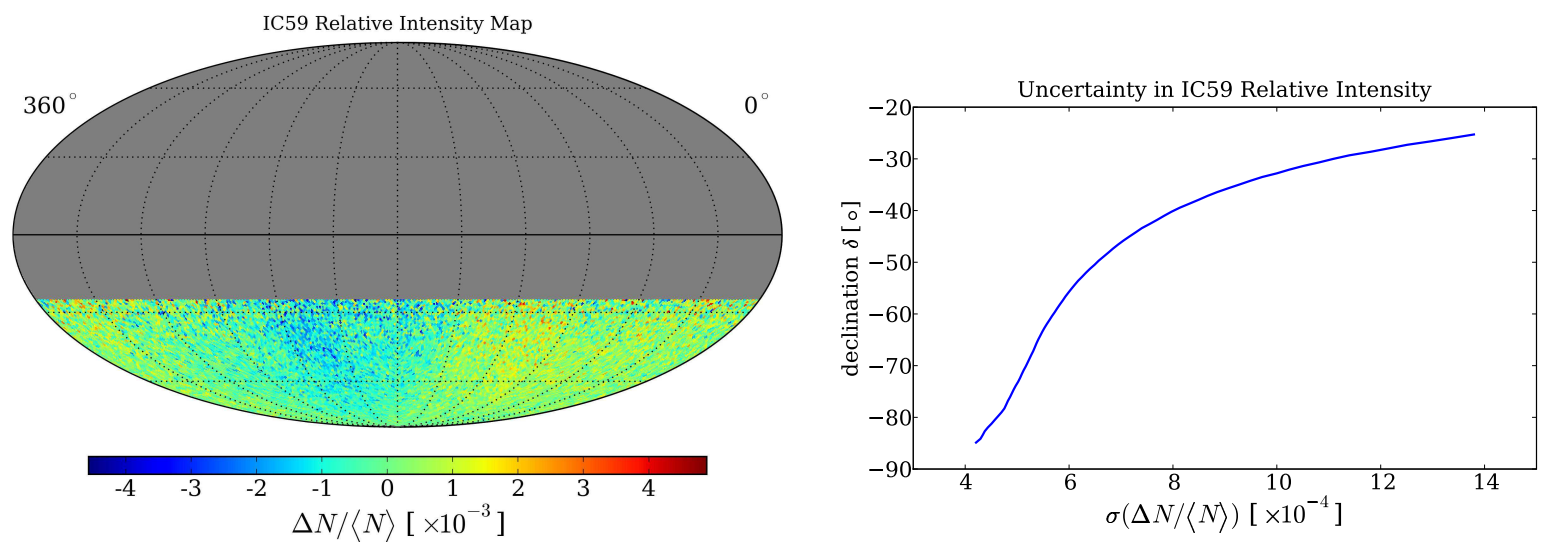

Fig. 2.- Left: Relative intensity $\Delta N /\langle N\rangle$ of the IC59 data in equatorial coordinates, produced with a time window of 24 hours. Right: Dependence of the statistical error on the declination.

which gives the amplitude of deviations from the isotropic expectation in each angular bin $i$. The deviations from isotropy can also be expressed in terms of a statistical significance using the method of Li \& Ma (1983). We report both relative intensity maps and significance maps in this paper.

The analyses in this paper use the HEALPix 2 library for the production of skymaps (Gorski et al. 2005). HEALPix produces an equal-area division of the unit sphere with pixels of roughly equal shape. The resolution of the HEALPix grid is defined by a parameter called $N_{\text {side }}$, which is related to the number of pixels in the grid by $N_{\text {pix }}=12 N_{\text {side }}^{2}$. Here, $N_{\text {side }}=64$ has been chosen, so that the sky is divided into 49152 pixels with an average pixel size of about $0.9^{\circ}$. Due to the zenith angle cut of $65^{\circ}$ discussed in Section 2.2, the pixels above declination $\delta=-25^{\circ}$ are masked in the analysis. This leaves 14196 pixels in the region between $\delta=-25^{\circ}$ and the celestial South Pole at $\delta=-90^{\circ}$. The sky maps are plotted in equatorial coordinates using an equal-area homolographic projection.

Figure2 (left) shows the relative intensity when a 24-hour time window is used to estimate the reference level. The map exhibits clear structures. The most obvious features are a broad excess in the relative counts near right ascension $105^{\circ}$, and a broad deficit near $225^{\circ}$. The relative intensity in the excess (and deficit) region is of order $10^{-3}$. This structure is the large-scale anisotropy first observed in the analysis of the IC22 data set and reported in Abbasi et al. (2010b). Since the IC59 data set is larger than the IC22 data set by an order of magnitude, it is now possible to see the large scale structure directly in the data without further rebinning or averaging over many pixels.

Figure 2 (right) shows the statistical error on the relative intensity map. Relative intensity

\footnotetext{
${ }^{2}$ Hierarchical Equal-Area isoLatitude Pixelization of the sphere: http://healpix.jpl.nasa.gov
} 

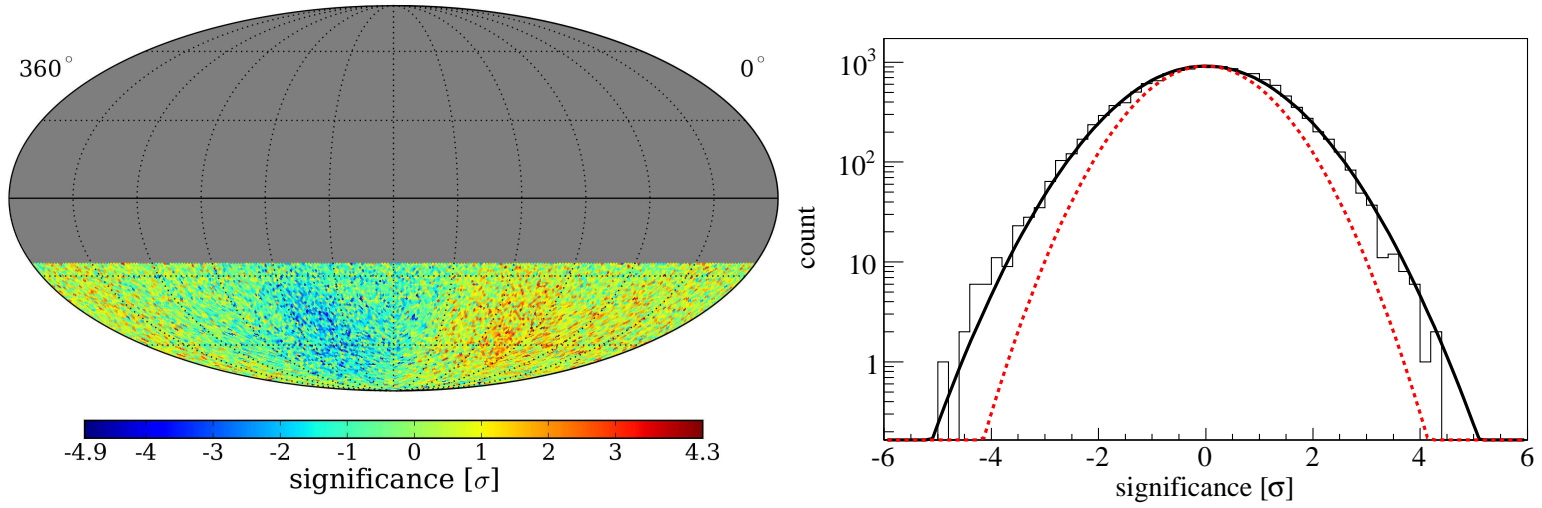

Fig. 3.- Left: Significance sky map of the IC59 data in equatorial coordinates, produced using a time window of 24 hours. Right: 1d-distribution of the significance values together with the best-fit (black solid line) performed with a Gaussian function. For comparison, a Gaussian function of mean zero and unit variance (red dashed line), expected from an isotropic sky, has been superimposed.

skymaps have declination-dependent statistical uncertainties due to the fact that the detector acceptance decreases with larger zenith angle. Since IceCube is located at the South Pole, the relative intensity exhibits large fluctuations near the horizon, corresponding to declinations $\delta>$ $-30^{\circ}$. Such edge effects are not as severe for skymaps of the significance of the fluctuations, though one must note that the location of structures with large (or small) significance may not coincide with regions of large (or small) relative intensity.

Fig. 3 (left) shows the significance map corresponding to the relative intensity map shown in Figure 2. The right panel also shows a distribution of the significance values in each bin. In an isotropic skymap, the distribution of the significance values should be normal (red dashed line). However, the best Gaussian fit to the distribution (black solid line) exhibits large deviations from a normal distribution caused by the large-scale structure.

\subsection{Angular Power Spectrum Analysis}

To observe correlations between pixels at several angular scales, we calculate the angular power spectrum of the relative intensity map $\delta I=\Delta N /\langle N\rangle$ described in Section 3.2. The relative intensity can be treated as a scalar field which we expand in terms of a spherical harmonic basis,

$$
\delta I\left(\mathbf{u}_{i}\right)=\sum_{\ell=1}^{\infty} \sum_{m=-\ell}^{\ell} a_{\ell m} Y_{\ell m}\left(\mathbf{u}_{i}\right)
$$




$$
a_{\ell m} \sim \Omega_{p} \sum_{i=0}^{N_{\mathrm{pix}}} \delta I\left(\mathbf{u}_{i}\right) Y_{\ell m}^{*}\left(\mathbf{u}_{i}\right) .
$$

In Eqs. (2) and (3), the $Y_{\ell m}$ are the Laplace spherical harmonics, the $a_{\ell m}$ are the multipole coefficients of the expansion, $\Omega_{p}$ is the solid angle observed by each pixel (which is constant across the sphere in HEALPix $), \mathbf{u}_{i}=\left(\alpha_{i}, \delta_{i}\right)$ is the pointing vector associated with the $i^{\text {th }}$ pixel, and $N_{\text {pix }}$ is the total number of pixels in the skymap. The power spectrum for the relative intensity field is defined as the variance of the multipole coefficients $a_{\ell m}$,

$$
\mathcal{C}_{\ell}=\frac{1}{2 \ell+1} \sum_{m=-\ell}^{\ell}\left|a_{\ell m}\right|^{2} .
$$

The amplitude of the power spectrum at some multipole order $\ell$ is associated with the presence of structures in the sky at angular scales of about $180^{\circ} / \ell$. In the case of complete and uniform sky coverage, a straightforward Fourier decomposition of the relative intensity maps would yield an unbiased estimate of the power spectrum. However, due to the limited exposure of the detector, we only have direct access to the so-called pseudo-power spectrum, which is the convolution of the real underlying power spectrum and the power spectrum of the relative exposure map of the detector in equatorial coordinates. In the case of partial sky coverage, the standard $Y_{\ell m}$ spherical harmonics do not form an orthonormal basis that we can use to expand the relative intensity field directly. As a consequence of this, the pseudo-power spectrum displays a systematic correlation between different $\ell$ modes that needs to be corrected for.

The deconvolution of pseudo-power spectra has been a longstanding problem in CMB astronomy, and there are several computationally efficient tools available from the CMB community. (For a discussion on the bias introduced by partial sky coverage in power spectrum estimation and a description of several bias removal methods, see Ansari \& Magneville (2010).) To calculate the power spectrum of the IC59 data, we use the publicly available Polspice 3 software package (Szapudi et al. 2001; Chon et al. 2004).

In PolSpice, the correction for partial sky bias is performed not on the power spectrum itself, but on the two-point correlation function of the relative intensity map. The two-point correlation function $\xi(\eta)$ is defined as

$$
\xi(\eta)=\left\langle\delta I\left(\mathbf{u}_{i}\right) \delta I\left(\mathbf{u}_{j}\right)\right\rangle,
$$

where $\delta I\left(\mathbf{u}_{k}\right)$ is the observed relative intensity in the direction of the $k^{\text {th }}$ pixel. Note that $\xi(\eta)$ depends only on the angle $\eta$ between any two pixels. The two-point correlation function can be expanded into a Legendre series,

$$
\xi(\eta)=\frac{1}{4 \pi} \sum_{\ell=0}^{\infty}(2 \ell+1) \mathcal{C}_{\ell} P_{\ell}(\cos \eta)
$$

\footnotetext{
${ }^{3}$ PolSpice website: http://prof.planck.fr/article141.html.
} 
where the $\mathcal{C}_{\ell}$ are the coefficients of the angular power spectrum and the $P_{\ell}$ are the Legendre polynomials. The inverse operation

$$
\mathcal{C}_{\ell}=2 \pi \int_{-1}^{1} \xi(\eta) P_{\ell}(\cos \eta) d(\cos \eta)
$$

can be used to calculate the angular power spectrum coefficients from a known two-point correlation function.

In order to obtain an unbiased estimator of the true power spectrum, PolSpice first calculates the $a_{\ell m}$ coefficients of both the relative intensity map and the relative exposure map doing a spherical harmonics expansion equivalent to that shown in Eq.(3). Pseudo-power spectra for both maps are computed from these coefficients using Eq.(4), and these spectra are subsequently converted into correlation functions using Eq.(6) . An unbiased estimator $\tilde{\xi}(\eta)$ of the true correlation function of the data is computed by taking the ratio of the correlation functions of the relative intensity map and the relative exposure map. An estimate $\tilde{\mathcal{C}}_{\ell}$ of the true power spectrum can then be obtained from the corrected two-point correlation function using the integral expression shown in Eq.(77).

This process reduces the correlation between different $\ell$ modes introduced by the partial sky coverage. Minor ringing artifacts associated with the limited angular range over which the correlation function is evaluated are minimized by applying an apodization function to the correlation function in $\eta$-space as described in Chon et al. (2004). The cosine apodization scheme provided by PolSpice and used in this work allows the correlation function to fall slowly to zero at large angular scales where statistics are low, minimizing any ringing artifacts that could arise from the calculation of the power spectrum from the corrected correlation function using Eq.(7).

Fig. 4 (blue points) shows the angular power spectrum for the IC59 relative intensity map from Fig.2. In addition to a strong dipole and quadrupole moment $(\ell=1,2)$, higher order terms up to $\ell=12$ contribute significantly to the skymap. The error bars on the $\tilde{\mathcal{C}}_{\ell}$ are statistical. The gray bands indicate the $68 \%$ and $95 \%$ spread in the $\tilde{\mathcal{C}}_{\ell}$ for a large number of power spectra for isotropic data sets (generated by introducing Poisson fluctuations in the reference skymap). As the $\tilde{\mathcal{C}}_{\ell}$ are still not entirely independent (even after the correction for partial sky coverage is performed), a strong dipole moment in the data can lead to significant higher order multipoles, and it is important to study whether the structure for $3 \leq \ell \leq 12$ is a systematic effect caused by the strong lower order moments $\ell=1,2$. Fig. 4 (red points) shows the angular power spectrum after the strong dipole and quadrupole moments are removed from the relative intensity map by a fit procedure described in the next section. The plot illustrates that after the removal of the lower order multipoles, indicated by the drop in $\tilde{\mathcal{C}}_{\ell}$ for $\ell=1,2$ (both are consistent with 0 after the subtraction), most of the higher order terms are still present. Only the strength of $\tilde{\mathcal{C}}_{3}$ and $\tilde{\mathcal{C}}_{4}$ is considerably reduced (the former to a value that is below the range of the plot).

Regarding systematic uncertainties, for $\ell=3$ and $\ell=4$ the effects of the strong dipole and quadrupole suggest that there is significant coupling between the low- $\ell$ modes. Therefore, we cannot rule out that $\tilde{\mathcal{C}}_{3}$ and $\tilde{\mathcal{C}}_{4}$ are entirely caused by systematic effects. For the higher multipoles, 


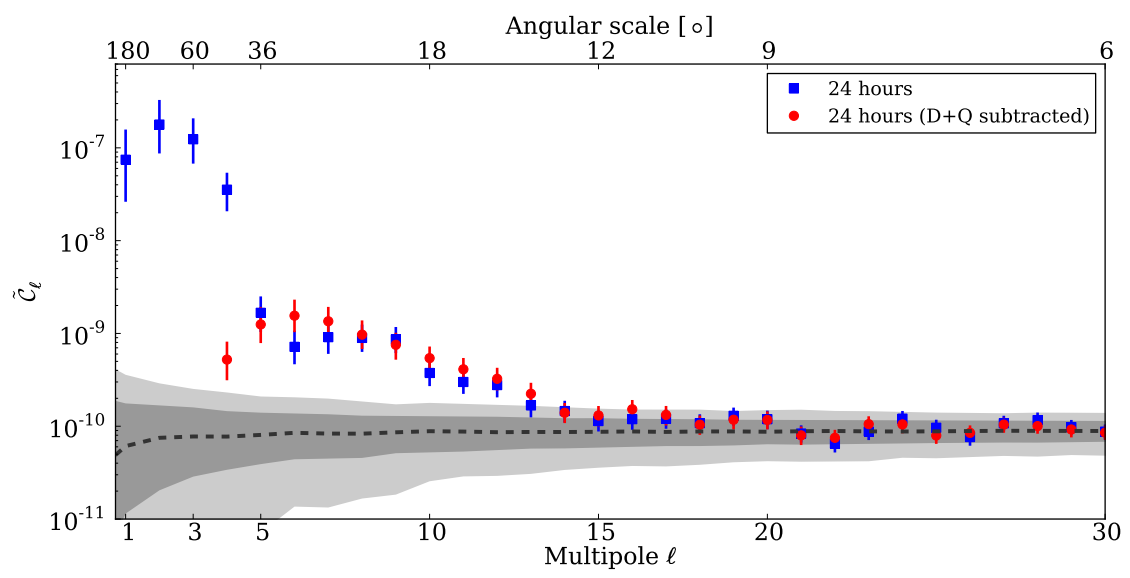

Fig. 4.- Angular power spectra for the relative intensity map shown in Fig.2, The blue and red points show the power spectrum before and after the subtraction of the dominant dipole and quadrupole terms from the relative intensity map. Errors bars are statistical, but a possible systematic error is discussed in the text. The gray bands indicate the distribution of the power spectra in a large sample of isotropic data sets, showing the $68 \%$ (dark) and $95 \%$ (light) spread in the $\tilde{\mathcal{C}}_{\ell}$.

the systematic effects of this distortion are much lower. After explicit subtraction of the $\ell=1$ and $\ell=2$ terms the residual power spectrum agrees with the original power spectrum within the statistical uncertainties. Therefore, we conclude that the systematic uncertainties in these data points are, at most, of the same order as the statistical uncertainties.

In summary, the skymap of cosmic ray arrival directions contains significant structures on scales down to $\sim 15^{\circ}$. In the next sections, we describe analysis techniques to make the smaller scale structure visible in the presence of the much stronger dipole and quadrupole moments.

\subsection{Subtraction of the Dipole and Quadrupole Moments}

A straightforward approach to understand the contribution of higher order multipoles and the corresponding structure in the skymap is to remove the strong dipole and quadrupole moments from the relative intensity map and study the residuals. This requires a dipole and quadrupole fit to the IC59 map. Once fit, the dipole and quadrupole can be subtracted from the skymap. We fit the relative intensity map using the function

$$
\begin{aligned}
& \delta I(\alpha, \delta)=m_{0}+p_{x} \cos \delta \cos \alpha+p_{y} \cos \delta \sin \alpha+p_{z} \sin \delta \\
& \quad+\frac{1}{2} Q_{1}\left(3 \cos ^{2} \delta-1\right)+Q_{2} \sin 2 \delta \cos \alpha+Q_{3} \sin 2 \delta \sin \alpha+Q_{4} \cos ^{2} \delta \cos 2 \alpha+Q_{5} \cos ^{2} \delta \sin 2 \alpha .
\end{aligned}
$$

Equation (8) is a multipole expansion of the relative count distribution in terms of real-valued spherical harmonic functions, and follows a normalization convention commonly used in CMB 


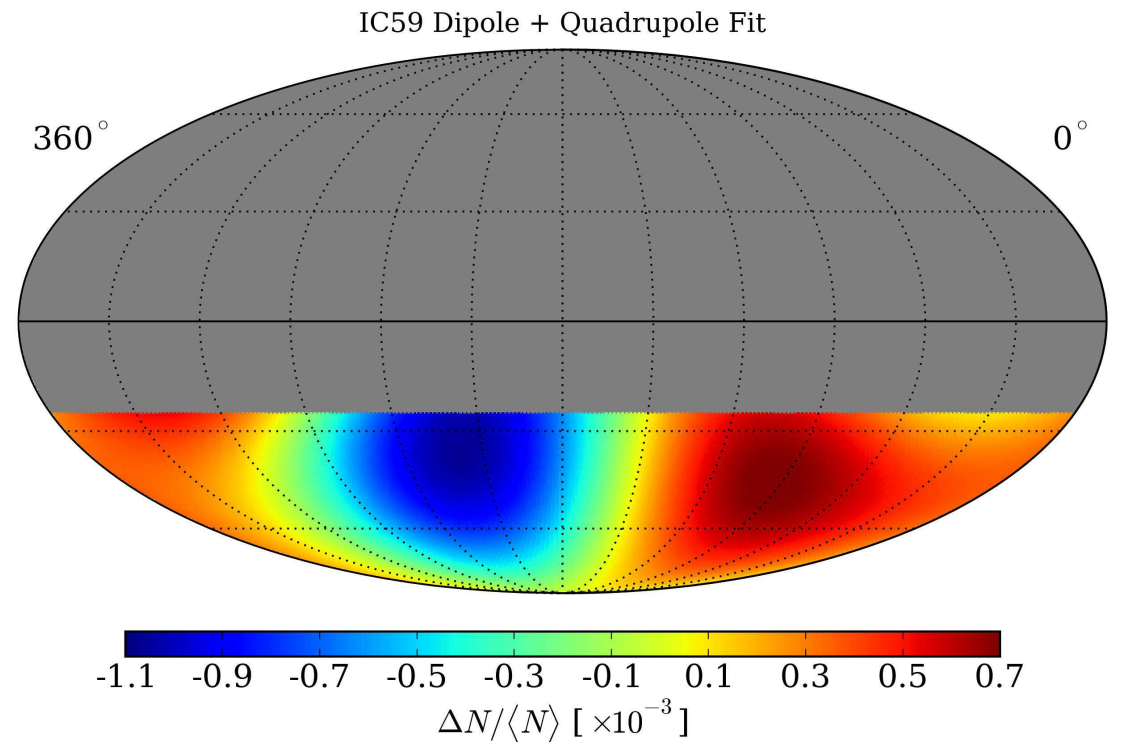

Fig. 5.- Fit of Eq. (8) to the IC59 relative intensity distribution $\Delta N /\langle N\rangle$ shown in Fig. 2 .

physics (Smoot \& Lubin 1979). The quantity $m_{0}$ is the "monopole" moment of the distribution, and corresponds to a constant offset of the data from zero. The values $\left(p_{x}, p_{y}, p_{z}\right)$ are the components of the dipole moment, and the quantities $\left(Q_{1}, \ldots, Q_{5}\right)$ are the five independent components of the quadrupole moment.

The two-dimensional harmonic expansion of Eq. (8) was fit to the 14196 pixels in the IC59 relative intensity map that lie between the celestial South Pole and declination $\delta=-25^{\circ}$. The best-fit dipole and quadrupole coefficients are provided in Table 1, and the corresponding sky distribution is shown in Fig. 5. By themselves, the dipole and quadrupole terms can account for much of the amplitude of the part-per-mille anisotropy observed in the IceCube data. We note that the quadrupole moment is actually the dominant term in the expansion, with a total amplitude that is about 2.5 times larger than the dipole magnitude. However, the $\chi^{2} / \mathrm{ndf}=14743 / 14187$ corresponds to a $\chi^{2}$-probability of approximately $0.05 \%$, so while the dipole and quadrupole are dominant terms in the arrival direction anisotropy, they do not appear to be sufficient to explain all of the structures observed in the angular distribution of $\Delta N /\langle N\rangle$. This result is consistent with the result of the angular power spectrum analysis in Sec. 3.3, which also indicates the need for higher-order multipole moments to describe the structures in the relative intensity skymap.

Subtraction of the dipole and quadrupole fits from the relative intensity map shown in Fig. 2 yields the residual map shown in Fig. 6. The fit residuals are relatively featureless at first glance, and the significance values are well-described by a normal distribution, which is expected when no anisotropy is present. However, the bin size in this plot is not optimized for a study of significant 


\begin{tabular}{|c|c|c|c|c|c|c|c|c|c|c|}
\hline \multirow{2}{*}{$\begin{array}{c}\text { Coefficient } \\
m_{0}\end{array}$} & \multirow{2}{*}{$\begin{array}{c}\text { Value (stat. + syst.) } \\
\left(\times 10^{-4}\right) \\
0.32 \pm 2.26 \pm 0.28\end{array}$} & \multicolumn{9}{|c|}{$\begin{array}{c}\text { Correlation Coefficients } \\
\chi^{2} / \mathrm{ndf}=14743 / 14187: \operatorname{Pr}\left(\chi^{2} \mid \mathrm{ndf}\right)=5.5 \times 10^{-4}\end{array}$} \\
\hline & & 1.00 & & & & & & & & \\
\hline$p_{x}$ & $2.44 \pm 0.71 \pm 0.30$ & 0.00 & 1.00 & & & & & & & \\
\hline$p_{y}$ & $-3.86 \pm 0.71 \pm 0.94$ & 0.00 & 0.00 & 1.00 & & & & & & \\
\hline$p_{z}$ & $0.55 \pm 3.87 \pm 0.45$ & 1.00 & 0.00 & 0.00 & 1.00 & & & & & \\
\hline$Q_{1}$ & $0.23 \pm 1.70 \pm 0.17$ & 0.99 & 0.00 & 0.00 & 0.99 & 1.00 & & & & \\
\hline$Q_{2}$ & $-2.95 \pm 0.49 \pm 0.74$ & 0.00 & 0.98 & 0.00 & 0.00 & 0.00 & 1.00 & & & \\
\hline$Q_{3}$ & $-8.80 \pm 0.49 \pm 0.50$ & 0.00 & 0.00 & 0.98 & 0.00 & 0.00 & 0.00 & 1.00 & & \\
\hline$Q_{4}$ & $-2.15 \pm 0.20 \pm 0.50$ & 0.00 & 0.00 & 0.00 & 0.00 & 0.00 & 0.00 & 0.00 & 1.00 & \\
\hline$Q_{5}$ & $-5.27 \pm 0.20 \pm 0.06$ & 0.00 & 0.00 & 0.00 & 0.00 & 0.00 & 0.00 & 0.00 & 0.00 & 1.00 \\
\hline
\end{tabular}

Table 1: Coefficients for the fit of Eq. (8) to the IC59 relative intensity distribution. The correlation coefficients indicate that there is some degeneracy between the contributions of $p_{x}$ and $Q_{2}, p_{y}$ and $Q_{3}$, and $p_{z}$ and $Q_{1}$ due to the fact that the IceCube detector only has a partial view of the sky. The systematic error on the fit parameters is estimated using the results of a fit using anti-sidereal time as described in Sec. 4.2,

anisotropy at angular scales larger than the angular resolution of the detector. To improve the sensitivity to larger features, we apply a smoothing procedure which simply takes the reference level and residual data counts in each bin and adds the counts from pixels within some angular radius of the bin. This procedure results in a map with Poisson uncertainties, though the bins are no longer statistically independent.

The actual size of any possible excess or deficit region (and thus the optimal smoothing scale) is not known a priori. Furthermore, the skymap may contain several significant structures of different size, with the optimal smoothing radius differing for each structure. To make the search as comprehensive as possible, we study the skymap on all smoothing scales from $3^{\circ}$ (the angular resolution) to $45^{\circ}$ in steps of $1^{\circ}$ and search for regions of high significance at any location. Applying this procedure, the two most significant localized excesses on the sky are a region with a peak significance of $7.0 \sigma$ at a smoothing radius of $22^{\circ}$ at $\left(\alpha=122.4^{\circ}, \delta=-47.4^{\circ}\right)$, and a region of peak significance $6.7 \sigma$ at a smoothing radius of $13^{\circ}$ at $\left(\alpha=263.0^{\circ}, \delta=-44.1^{\circ}\right)$. These values do not account for statistical trials due to the scan over smoothing radii or the scan for the peak significance in the 14196 pixels. We have estimated the trial factors by applying the same search strategy to a large number of simulated isotropic data sets. After trial factors are applied, the maximum significance of the "hot spot" with an optimal smoothing radius of $22^{\circ}$ is reduced to $5.3 \sigma$, and the "hot spot" at $13^{\circ}$ is reduced to $4.9 \sigma$.

Skymaps of the relative intensity and the significance of the residual data are plotted in Fig.7, where a smoothing radius of $20^{\circ}$ has been used. The radius is not optimal for any of the most significant excesses, but with this choice all of the significant features can be seen with reasonable resolution. Compared to the intensity of the dipole and quadrupole shown in Fig.2, the smaller 

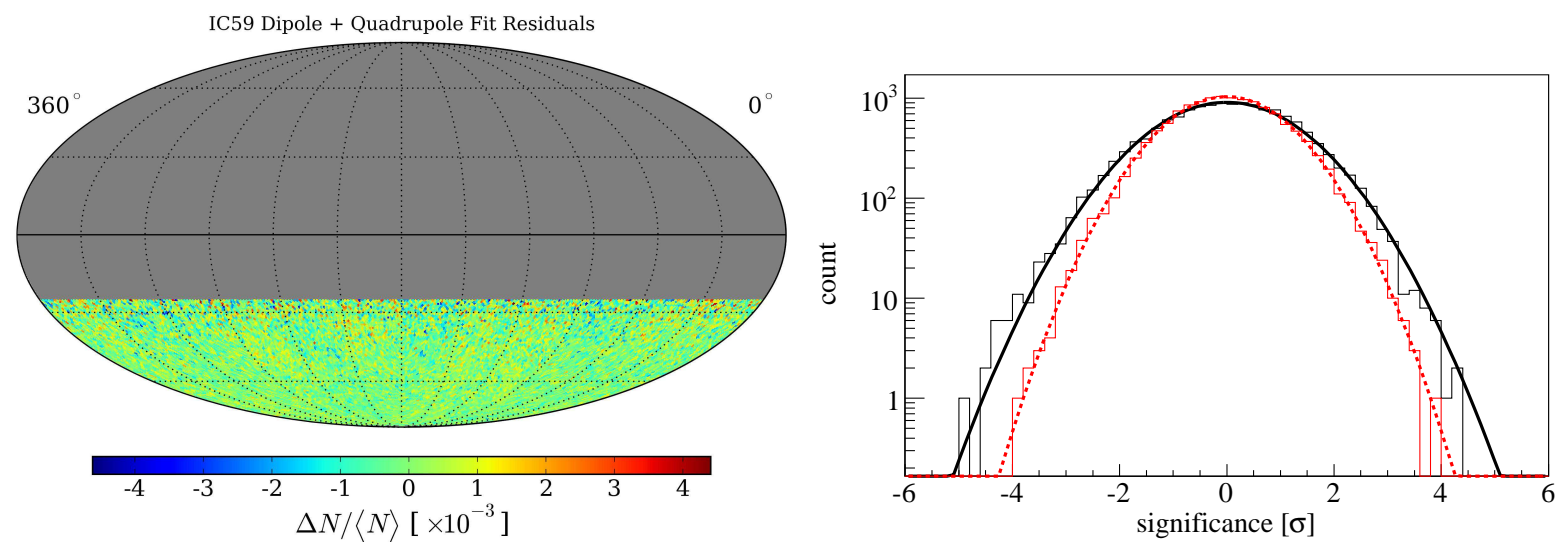

Fig. 6. - Left: Residual of the fit of Eq. (8) to the relative intensity distribution shown in Fig. 2, Right: Distribution of pixel significance values in the skymap before (solid black line) and after (dashed red line) subtraction of the dipole and quadrupole. Gaussian fits to the data yield a mean of $(-0.20 \pm 1.05) \times 10^{-2}$ and a width of $1.23 \pm 0.01$ before the dipole and quadrupole subtraction, and $(0.28 \pm 0.89) \times 10^{-2}$ and $1.02 \pm 0.01$ after.

structures are weaker by about a factor of 5 .

Table 2 contains the location and optimal smoothing scales of all the regions in the IC59 skymap which have a pre-trials significance beyond $\pm 5 \sigma$. The data also exhibit additional regions of excess and deficit. It is possible that the deficits are at least in part artifacts of the reference level estimation procedure, which can produce artificial deficits around regions of significant excess counts (or in principle, excesses in the presence of strong physical deficits). While several of the deficit and excess regions are observed at large zenith angles near the edge of the IC59 exposure region, we do not believe these features are statistical fluctuations or edge effects. As we will show in Section 4.3, the features are also present in IC22 and IC40 data, and grow in significance as the statistics increase.

Fig. 8 shows the significance maps with regions with a pre-trial significance larger than $\pm 5 \sigma$ indicated according to the numbers used in Table 2. Since the optimal scales vary from region to region and no single smoothing scale shows all regions, we show the maps with two smoothing scales, $12^{\circ}$ (left), and $20^{\circ}$ (right).

The angular power spectrum of the residual map is shown in red in Fig. 4. As expected, there is no significant dipole or quadrupole moment left in the skymap, and the $\ell=3$ and $\ell=4$ moments have also disappeared or have been weakened substantially. However, the moments corresponding to $5 \leq \ell \leq 12$ are still present at the same strength as before the subtraction, and indicate the presence of structure of angular size $15^{\circ}$ to $35^{\circ}$ in the data. The excesses and deficits in Fig. 7 

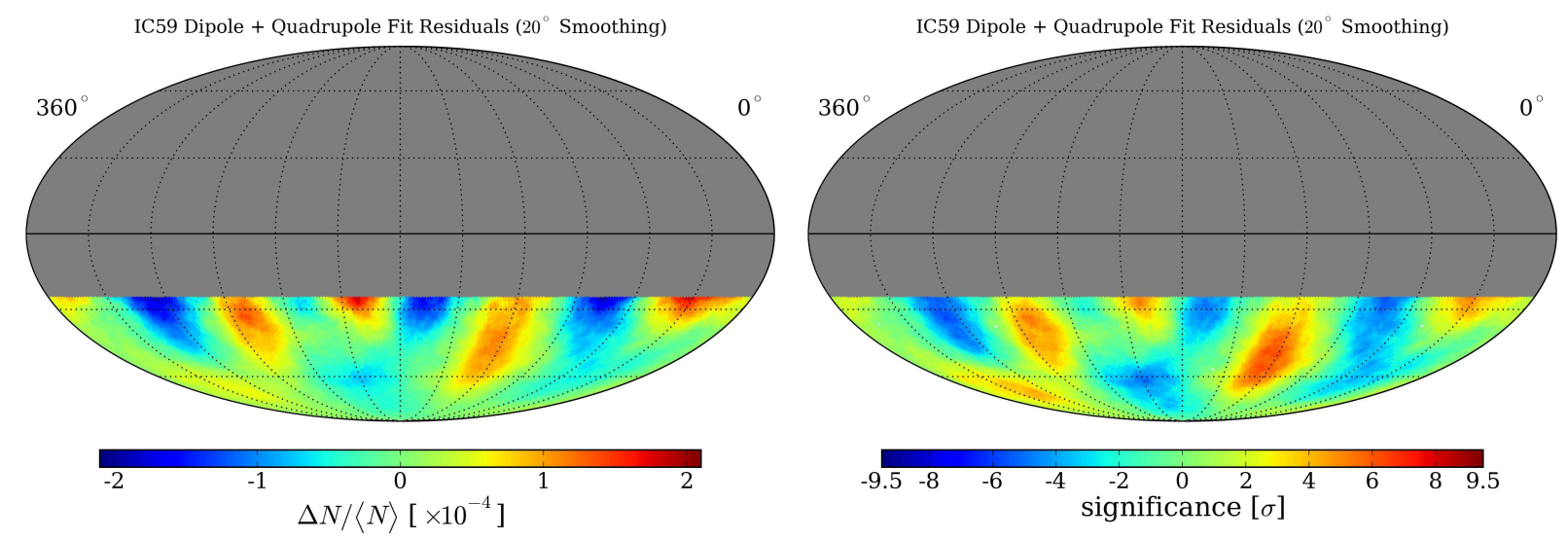

Fig. 7.- Left: Residual intensity map plotted with $20^{\circ}$ smoothing. Right: Significances of the residual map (pre-trials), plotted with $20^{\circ}$ smoothing.

correspond in size to these moments.

\begin{tabular}{cccccc}
\hline region & right ascension & declination & optimal scale & peak significance & post-trials \\
\hline 1 & $\left(122.4_{-4.7}^{+4.1}\right)^{\circ}$ & $\left(-47.4_{-3.2}^{+7.5}\right)^{\circ}$ & $22^{\circ}$ & $7.0 \sigma$ & $5.3 \sigma$ \\
2 & $\left(263.0_{-3.8}^{+3.7}\right)^{\circ}$ & $\left(-44.1_{-5.1}^{+5.3}\right)^{\circ}$ & $13^{\circ}$ & $6.7 \sigma$ & $4.9 \sigma$ \\
3 & $\left(201.6_{-1.1}^{+6.0}\right)^{\circ}$ & $\left(-37.0_{-1.9}^{+2.2}\right)^{\circ}$ & $11^{\circ}$ & $6.3 \sigma$ & $4.4 \sigma$ \\
4 & $\left(332.4_{-7.1}^{+9.5}\right)^{\circ}$ & $\left(-70.0_{-7.6}^{+4.2}\right)^{\circ}$ & $12^{\circ}$ & $6.2 \sigma$ & $4.2 \sigma$ \\
\hline 5 & $\left(217.7_{-7.8}^{+10.2}\right)^{\circ}$ & $\left(-70.0_{-2.3}^{+3.6}\right)^{\circ}$ & $12^{\circ}$ & $-6.4 \sigma$ & $-4.5 \sigma$ \\
6 & $\left(77.6_{-8.4}^{+3.9}\right)^{\circ}$ & $\left(-31.9_{-8.6}^{+3.2}\right)^{\circ}$ & $13^{\circ}$ & $-6.1 \sigma$ & $-4.1 \sigma$ \\
7 & $\left(308.2_{-7.7}^{+4.8}\right)^{\circ}$ & $\left(-34.5_{-6.9}^{+9.6}\right)^{\circ}$ & $20^{\circ}$ & $-6.1 \sigma$ & $-4.1 \sigma$ \\
8 & $\left(166.5_{-5.7}^{+4.5}\right)^{\circ}$ & $\left(-37.2_{-5.7}^{+5.0}\right)^{\circ}$ & $12^{\circ}$ & $-6.0 \sigma$ & $-4.0 \sigma$ \\
\hline
\end{tabular}

Table 2: Location and optimal smoothing scale for regions of the IC59 skymap with a pre-trials significance larger than $\pm 5 \sigma$. The errors on the equatorial coordinates indicate the range over which the significance drops by $1 \sigma$ from the local extremum. 
IC59 Dipole + Quadrupole Fit Residuals $\left(12^{\circ}\right.$ Smoothing $)$

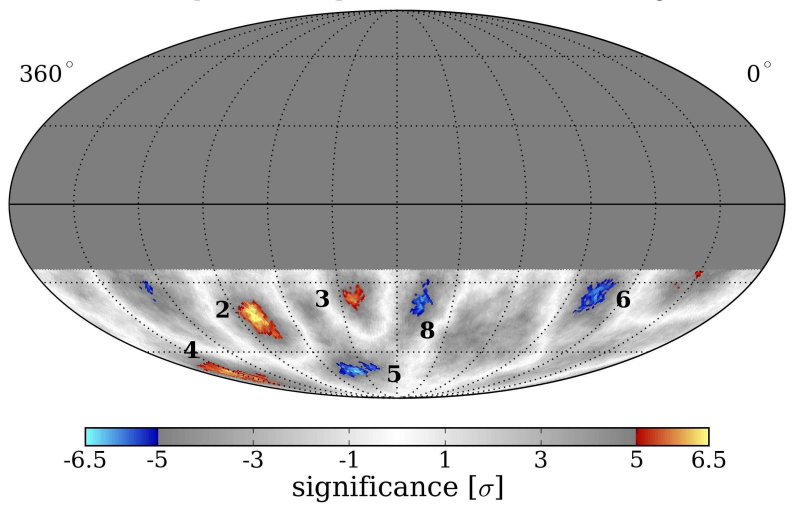

IC59 Dipole + Quadrupole Fit Residuals $\left(20^{\circ}\right.$ Smoothing $)$

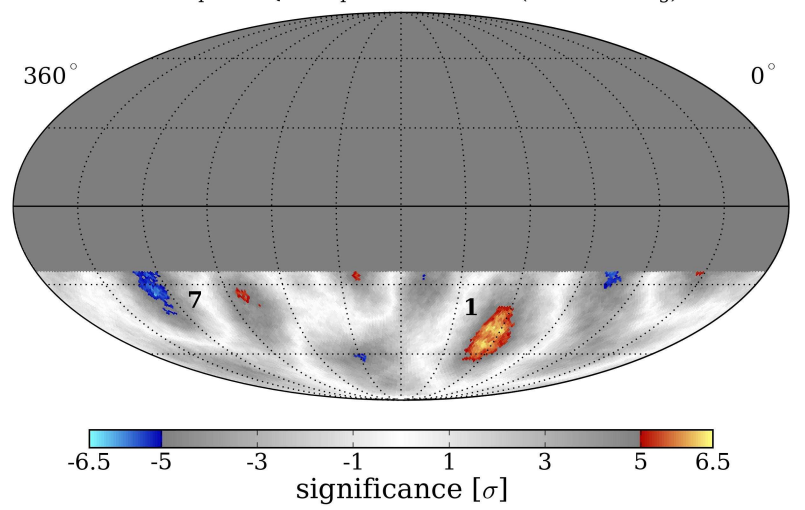

Fig. 8.- Left: Significances of the IC59 residual map plotted with $12^{\circ}$ smoothing. Right: Significances of the IC59 residual map plotted with $20^{\circ}$ smoothing. The regions with a pre-trial significance larger than $\pm 5 \sigma$ are indicated according to the numbers used in Table 2 . 


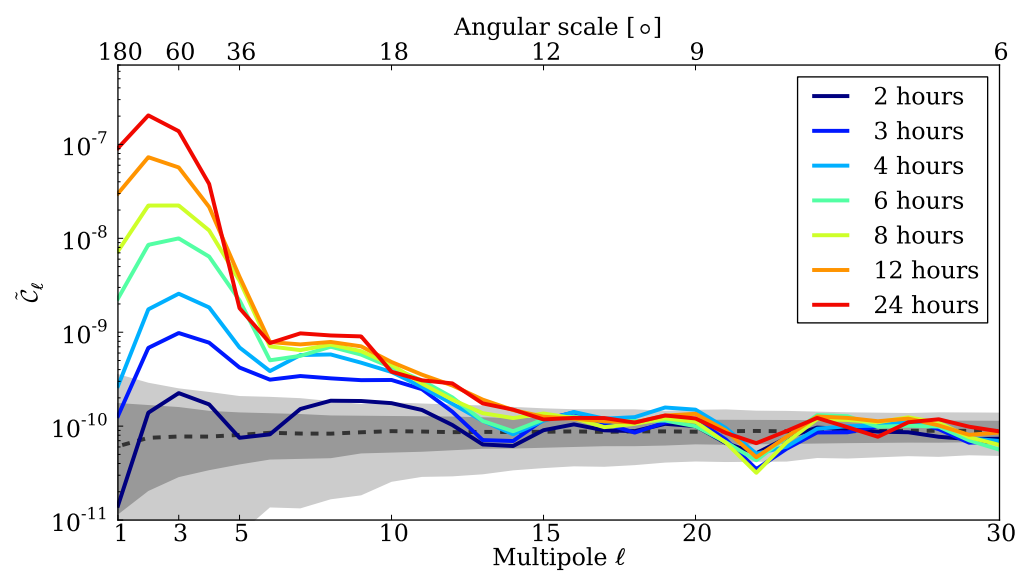

Fig. 9.- Power spectra for different values of the time scrambling period $\Delta t$. The filtering effect of the time scrambling on large-scale structure can be easily seen as a monotonic reduction in the strength of low- $\ell$ components of the power spectrum. The grey bands show 1 and 2 sigma bands for a large set of isotropic skymaps. See Fig. 4 and Section 3.3 for statistical uncertainties and a discussion of systematic uncertainties.

\subsection{A Filter for Structure on Small Angular Scales}

In previous works (Abdo et al. 2008; Vernetto et al. 2009), a different method is applied to filter the lower $\ell$ terms and create skymaps showing the small-scale structure. In these analyses, the dipole and quadrupole moments are not fit and subtracted, but suppressed by varying the time window $\Delta t$ over which the reference level is estimated (i.e., the length of time in which the time scrambling, or any other method for generating an isotropic sky, is performed). We apply this method to the IC59 data to compare the results to the dipole and quadrupole subtraction outlined in Sec.3.4.

Different time windows probe the presence of anisotropy at different angular scales. The time scrambling fits structures that are larger than $15^{\circ} /$ hour $\times \Delta t$, and the angular size of a multipole of order $\ell$ in the sky is $\sim 180^{\circ} / \ell$. This implies that the technique filters out modes with $\ell<12$ hours $/ \Delta t$ and reduces the magnitude of the modes near this threshold.

The efficiency of the method in suppressing larger structures (low- $\ell$ moments) is demonstrated in Fig.9, where the angular power spectra are plotted for relative intensity maps constructed with seven values of $\Delta t$ between 2 hours and 24 hours. As expected, the strength of the low-order multipoles decreases monotonically with $\Delta t$. However, the power spectrum also reveals that the low- $\ell$ moments, in particular the quadrupole term, are not completely removed from the data unless $\Delta t$ is as small as 3 hours. In addition, the choice of $\Delta t \leq 3$ hours also appears to weaken the power observed in the modes $3 \leq \ell \leq 12$. Consequently, the residual map from Sec. 3.4 and the skymaps produced by choosing a small $\Delta t$ cannot be expected to agree in all details. Nevertheless, 

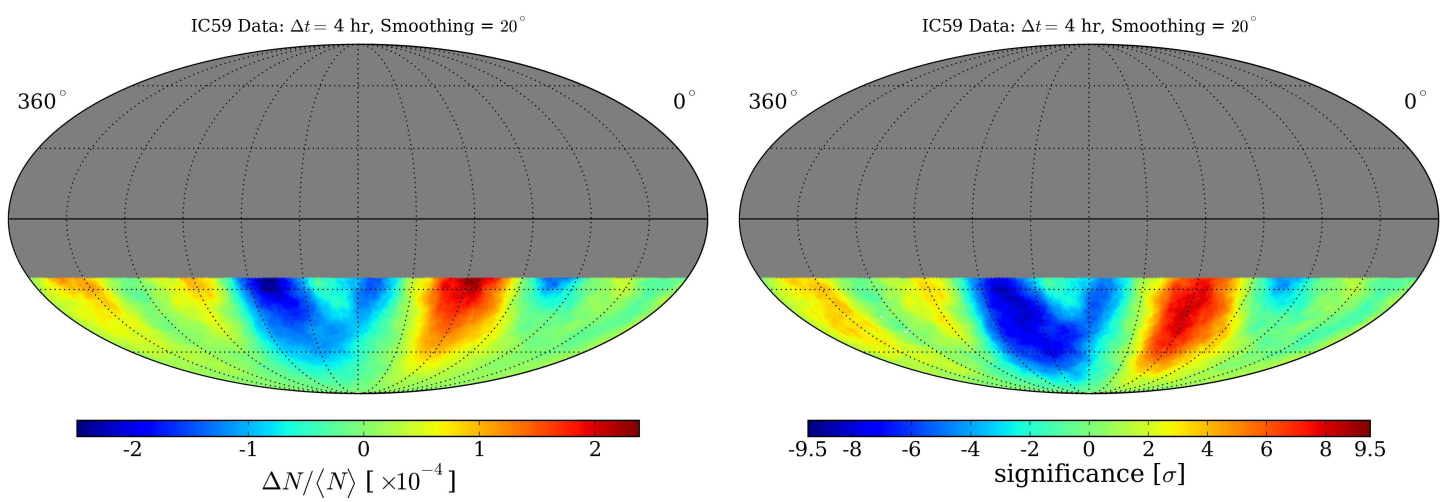

Fig. 10.- Relative intensity (left) and significance (right) map in equatorial coordinates for $\Delta t=$ 4 hours and an integration radius of $20^{\circ}$.

a comparison of the skymaps produced with the two methods provides an important crosscheck.

To best compare this analysis to the results of Section 3.4, the reference level is calculated using a scrambling time window of $\Delta t=4$ hours. This choice of $\Delta t$ is motivated by the angular power spectrum in Fig.9. With $\Delta t=4$ hours, the spectrum shows the strongest suppression of the dipole and quadrupole while still retaining most of power in the higher multipole moments.

Skymaps of the relative intensity and significance for $\Delta t=4$ hours are shown in Fig. 10. The maps have been smoothed by $20^{\circ}$ to allow for a direct comparison with Fig. 7 . The most prominent features of the map are a single broad excess and deficit, with several small excess regions observed near the edge of the exposure region. The broad excess is centered at $\alpha=\left(121.7_{-7.1}^{+4.8}\right)^{\circ}$ and $\delta=\left(-44.2_{-7.8}^{+12.1}\right)^{\circ}$, at the same position as Region 1 in Table 2. The optimal smoothing scale of the excess is $25^{\circ}$, with a pre-trials significance of $9.6 \sigma$. A second significant excess is observed at $\alpha=\left(341.7_{-5.6}^{+1.4}\right)^{\circ}$ and $\delta=\left(-34.9_{-6.8}^{+3.6}\right)^{\circ}$ with a peak significance of $5.8 \sigma$ at a smoothing scale of $9^{\circ}$. This feature does not appear to have a direct match in Fig. 17, but is roughly aligned in right ascension with the excess identified in Table 2 as Region 4 . We also note that the secondlargest excess in Table 2, Region 2, is visible near $\alpha=263.0^{\circ}$ in Fig. 10, but with a pre-trials peak significance of $4.5 \sigma$ after smoothing by $13^{\circ}$.

The differences in significance between Figs. 7 and 10 can be attributed to the fact that some contributions from the low- $\ell$ moments are still present in this analysis. The broad excess observed here is co-located with the maximum of the large-scale structure shown in Fig. 5, enhancing its significance. By comparison, the excess in Region 2 is close to the minimum of the large-scale structure, weakening its significance. The leakage of large-scale structure into the $\Delta t=4$ hour skymap also explains the large deficit near $\alpha=220^{\circ}$; due to its co-location with the minimum of the dipole and quadrupole, the size of the deficit is enhanced considerably. 


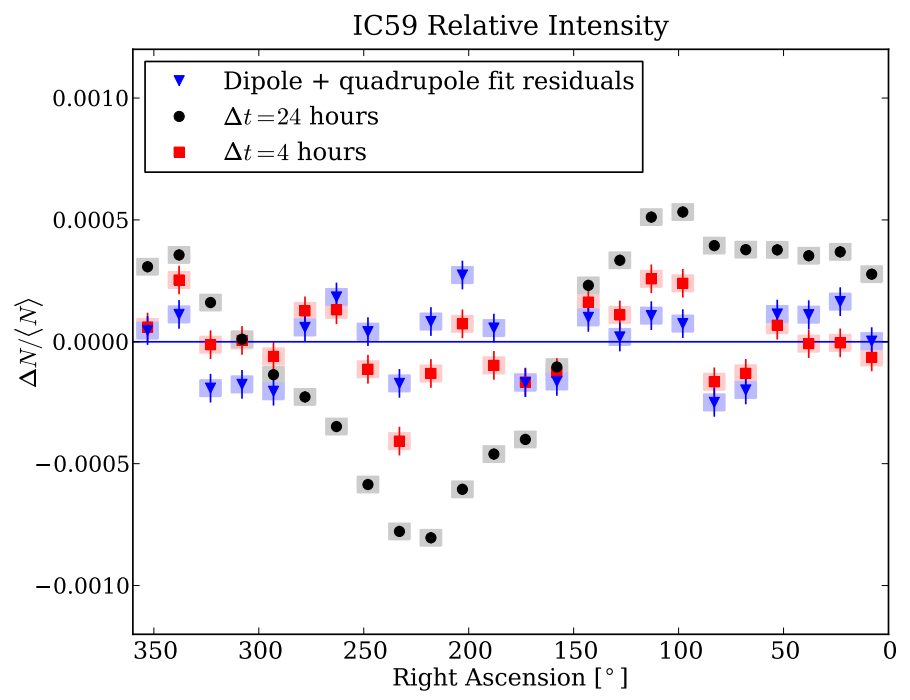

Fig. 11.- Relative intensity in the declination band $-45^{\circ}<\delta<-30^{\circ}$. The blue points show the result after subtracting the dipole and quadrupole moments. The black points correspond to $\Delta t$ $=24$ hours and show the large-scale structure, the red points correspond to $\Delta t=4$ hours. The error boxes represent systematic uncertainties.

This effect is illustrated in Fig.11, which shows the relative intensity for the declination range $-45^{\circ}<\delta<-30^{\circ}$, projected onto the right ascension axis. This declination range is chosen because it contains some of the most significant structures of the skymaps. The blue points show the relative intensity corresponding to Fig.7, i.e., the skymap after subtraction of dipole and quadrupole moments. The black and red points show the relative intensity for skymaps obtained with the method described in this section; the black points correspond to $\Delta t=24$ hours, the red points to $\Delta t=4$ hours. In the case of $\Delta t=24$ hours, the large scale structure dominates. For $\Delta t=4$ hours, the large scale structure is suppressed, and the smaller features become visible. The blue and red curves show excesses and deficits at the same locations, but with different strengths. As the red curve still contains some remaining large scale structure, maxima and minima are enhanced or weakened depending on where they are located with respect to the maximum and minimum of the large-scale structure. The systematic error for the relative intensity values in Fig.11 is taken from the analysis of the data in anti-sidereal time as described in the next section.

Finally, we note that the presence of the small-scale structure can be verified by inspection of the raw event counts in the data. Figure 12 shows the observed and expected event counts for declinations $-45^{\circ}<\delta<-30^{\circ}$, projected onto the right ascension axis. The seven panels of the figure contain the projected counts for seven time scrambling windows $\Delta t=\{2,3,4,6,8,12,24$ hours $\}$. For small values of $\Delta t$, the expected counts agree with the data; for example, when $\Delta t=2$ hours, the data exhibit no visible deviation from the expected counts. For larger values of $\Delta t$, the expected 
count distribution flattens out as the technique to estimate the reference level no longer over-fits the large structures. When $\Delta t=24$ hours, the reference level is nearly flat, and the shape of the large-scale anisotropy is clearly visible from the raw data. 

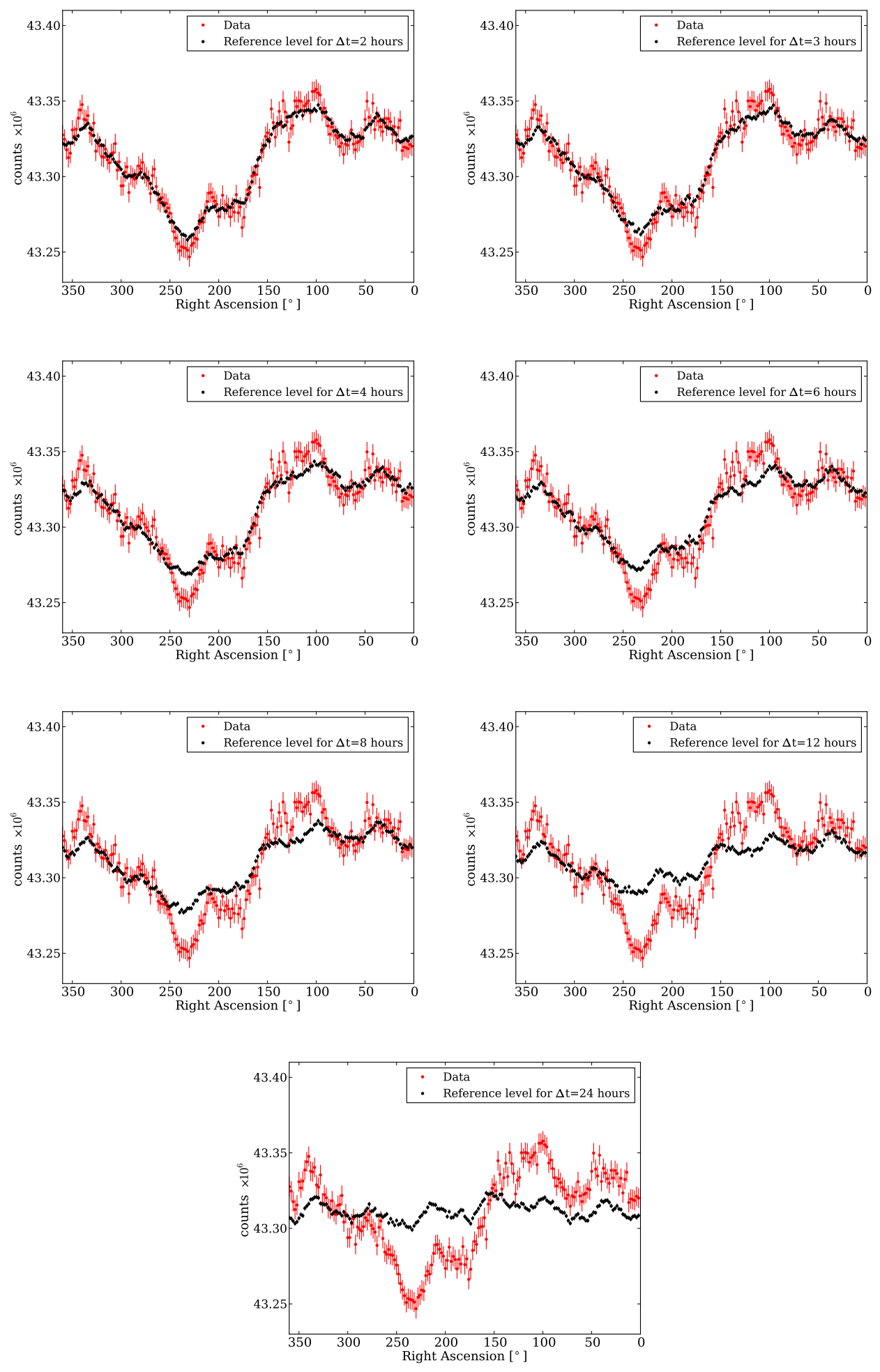

Fig. 12.- Number of events (red) and reference level (black), with statistical uncertainties, as a function of right ascension for the declination range $-45^{\circ}<\delta<-30^{\circ}$. The reference level is estimated in different time windows, from 2 hours (top-left) to 24 hours (bottom). Each plot has been created using independent $15^{\circ} \delta \times 2^{\circ}$ bins in right ascension. 


\section{Systematic Checks}

Several tests have been performed on the data to ensure the stability of the observed anisotropy and to rule out possible sources of systematic bias. Among the influences that might cause spurious anisotropy are the detector geometry, the detector livetime, nonuniform exposure of the detector to different regions of the sky, and diurnal and seasonal variations in atmospheric conditions. Due to the unique location of the IceCube detector at the South Pole, many of these effects play a lesser role for IceCube than for detectors located in the middle latitudes. The southern celestial sky is fully visible to IceCube at any time, and changes in the event rate tend to affect the entire visible

sky. Seasonal variations are of order $\pm 10 \%$ (Tilav et al. 2009), but the changes are slow and the reference level estimation technique is designed to take these changes into account. This is also true for any effects caused by the asymmetric detector response due to the geometrical configuration of the detector. In this section, we test the accuracy of these assumptions.

\subsection{Solar Dipole Analysis}

As mentioned in Section 1.1, any observer moving through a plasma of isotropic cosmic rays should observe a difference in intensity between the direction of the velocity vector and the opposite direction. Therefore, cosmic rays received on Earth should exhibit a dipole modulation in solar time caused by the Earth's orbital velocity around the Sun. The expected change in the relative intensity is given by

$$
\frac{\Delta I}{\langle I\rangle}=(\gamma+2) \frac{v}{c} \cos \rho
$$

where $I$ is the cosmic ray intensity, $\gamma=2.7$ the power law index of the cosmic ray energy spectrum, $v / c$ the ratio of the Earth's velocity with respect to the speed of light, and $\rho$ the angle between the cosmic ray arrival direction and the direction of motion (Gleeson \& Axford 1968). With a velocity of $v=30 \mathrm{~km} \mathrm{~s}^{-1}$, the expected amplitude is $4.7 \times 10^{-4}$. Note that the power law spectral index has a systematic uncertainty (see for example Biermann et al. (2010) for a discussion) and the Earth's velocity is not precisely constant, but both of these uncertainties are too small to be relevant in our comparison of the predicted dipole strength to the measured strength. The solar dipole effect has been measured with several experiments (Amenomori et al. 2004, 2008; Abdo et al. 2009) and provides an important check of the reliability of the analysis techniques presented earlier, as it verifies that the techniques are sensitive to a known dipole with an amplitude of roughly the same size as the structures in the equatorial skymap.

In principle, the solar dipole is not a cause of systematic uncertainties in the analysis of cosmic ray anisotropy in sidereal time (equatorial coordinates). The solar dipole is visible only when the arrival directions are plotted in a frame where the Sun's position is fixed in the sky. A signal in this coordinate system averages to zero in sidereal time over the course of one year. However, any seasonal variation of the solar dipole can cause a spurious anisotropy in equatorial coordinates. The effect works both ways: a seasonal variation in the sidereal anisotropy will affect the solar dipole. 


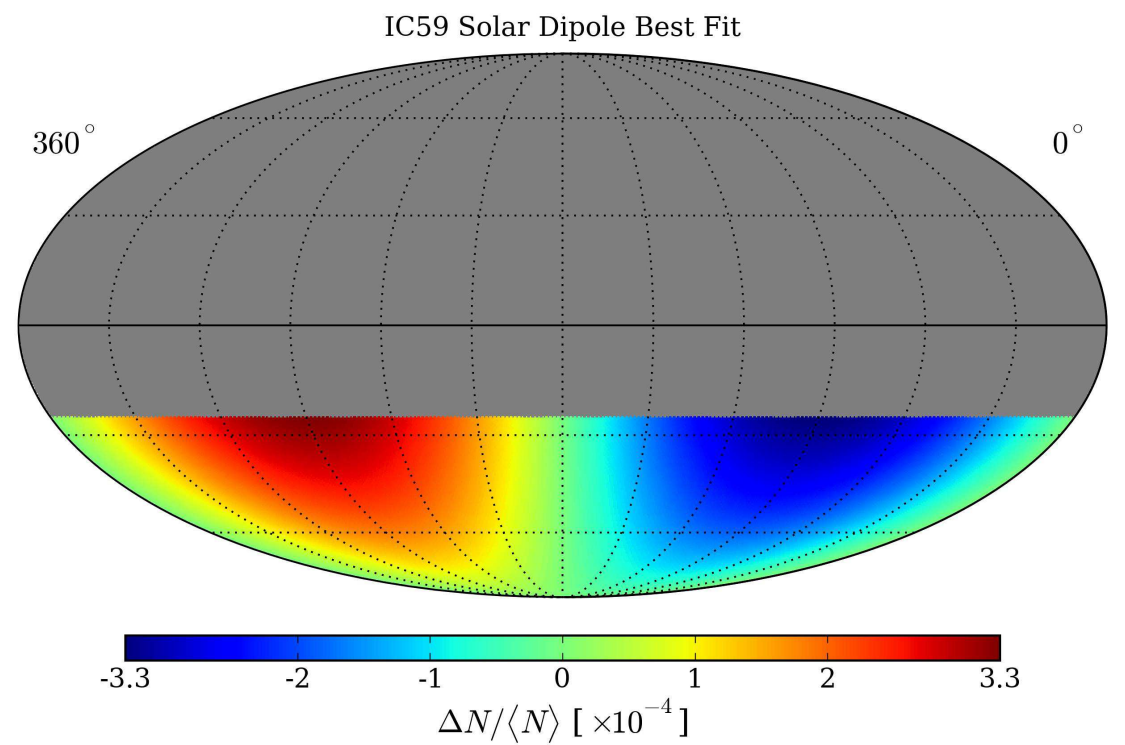

Fig. 13. - Best fit results to the IC59 data expressed in solar coordinates. In this coordinate system, the velocity vector of the motion of the Earth about the Sun is oriented at a longitude of $270^{\circ}$.

A standard way to study the extent of these contaminations is by use of two artificial time scales, anti-sidereal and extended-sidereal time. Anti-sidereal time is calculated by reversing the sign of the transformation between universal time and sidereal time. Each sidereal day is slightly shorter than the solar day (universal time) by about 4 minutes, while each anti-sidereal day is longer than a solar day by the same factor. Anti-sidereal time therefore has 364.25 days (i.e. complete revolutions in the coordinate frame) per year, one day less than the solar year (365.25 days) and two days less than the sidereal year (366.25 days). Similarly, each extended sidereal day is shorter than a sidereal day by about 4 minutes ( 8 minutes shorter than the solar day). Extended sidereal time has therefore 367.25 days per year. No physical phenomena are expected to occur in the anti-sidereal or in the extended-sidereal frame. However, systematic distortions in the sidereal anisotropy due to seasonal variations of the solar dipole will produce a "signal" in anti-sidereal time. Similarly, distortions in the solar dipole due to seasonal variations of the sidereal anisotropy will produce a "signal" in extended-sidereal time. We follow the example of Amenomori et al. (2008) and Abdo et al. (2009) and use anti-sidereal time for an estimate of the error from seasonal variations on the amplitude of the sidereal anisotropy, and extended-sidereal time to estimate the systematic error on the solar dipole amplitude.

To measure the solar dipole anisotropy we estimate the reference level using a time window $\Delta t=24$ hours, which maximizes the sensitivity to large-scale features. The data and reference maps are produced in a coordinate system where the latitude coordinate is declination and the 


\begin{tabular}{cr}
\hline Coefficient & \multicolumn{1}{c}{ Value (stat. + syst.) } \\
& \multicolumn{1}{c}{$\left(\times 10^{-4}\right)$} \\
\hline$m_{0}$ & $-0.03 \pm 0.06 \pm 0.02$ \\
$p_{x}$ & $0.02 \pm 0.14 \pm 0.97$ \\
$p_{y}$ & $-3.66 \pm 0.14 \pm 0.17$ \\
$p_{z}$ & $-0.03 \pm 0.07 \pm 0.01$ \\
\hline
\end{tabular}

Table 3: Coefficients of a dipole and constant offset fit to the IC59 solar coordinate data. The systematic error on the fit parameters is estimated using the results of a fit using extended-sidereal time as described in the text.

longitude coordinate represents the angular distance from the Sun in right ascension, defined as the difference between the right ascension of each event and the right ascension of the Sun. In this coordinate system the Sun's longitude is fixed at $0^{\circ}$ and we expect, over a full year, an excess in the direction of motion of the Earth's velocity vector (at $270^{\circ}$ ) and a minimum in the opposite direction.

The data are fit using the dipole and quadrupole expansion given in Eq. (8). The quadrupole coefficients are found to be equivalent to zero within the fit uncertainties, so the fit is repeated with only a dipole term and a constant offset. The dipole describes the data well; the fit $\chi^{2} / \mathrm{ndf}=$ $14207 / 14192$ corresponds to a $\chi^{2}$-probability of $41.6 \%$. The best fit coefficients are listed in Table 3 , Only one free parameter, the $p_{y}$ component of the dipole fit, differs significantly from zero. Hence, the dipole is aligned at a longitude of $270^{\circ}$ within the equatorial plane of this coordinate system, following the expectation for a dipole in the cosmic ray skymap caused by relative motion about the Sun.

The amplitude of the dipole is $\left(3.66 \pm 0.14_{\text {stat }} \pm 0.99_{\text {sys }}\right) \times 10^{-4}$. The systematic uncertainty is evaluated by fitting a dipole to the data in a coordinate system using extended-sidereal time. We have conservatively estimated this systematic uncertainty by taking the amplitude of the dipole in extended-sidereal coordinates. Within the large systematic error, the amplitude of the solar dipole agrees with the prediction. A more detailed study of the solar dipole anisotropy in IceCube data will follow in a separate publication.

\subsection{Anti-Sidereal Time Analysis}

As described in the previous section, we use the analysis of the data in the anti-sidereal time frame to study systematic effects caused by seasonal variations. For this test, we produce skymaps where anti-sidereal time is used instead of sidereal time in the coordinate transformation from local detector coordinates to "equatorial" coordinates. Skymaps produced in this way are subjected to the same analyses as the true equatorial maps. Neither the angular power spectrum nor the skymaps show any significant deviation from isotropy. In particular, no regions of significant excess 

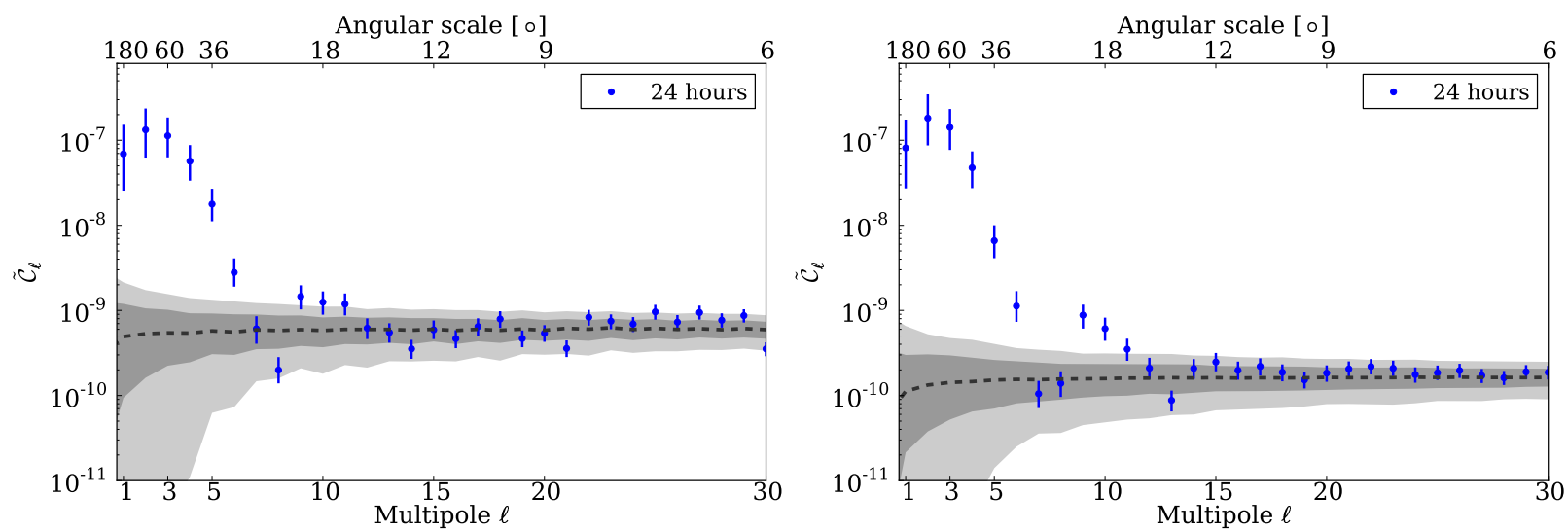

Fig. 14.- Angular power spectra for the relative intensity maps from IC22 (left), and IC40 data (right). Errors bars are statistical. The gray bands indicate the distribution of the power spectra in a large sample of isotropic data sets, showing the $68 \%$ (dark) and $95 \%$ (light) spread in the $\tilde{\mathcal{C}}_{\ell}$.

or deficit are observed in the anti-sidereal skymaps for any smoothing scale. The systematic error bars shown in Fig.11 are estimated by using the variation in anti-sidereal time as a measure of this error.

\subsection{Comparison with IC22 and IC40}

An important cross-check of the structure seen in the IC59 data set can be made by applying the IC59 analysis to data recorded in the two data periods prior to IC59. The IC22 data set contains 5 billion events recorded between July 2007 and April 2008, and the IC40 data set contains 19 billion events recorded between April 2008 and May 2009. While these data sets are smaller than the IC59 data set due to the smaller detector size, we nevertheless expect to observe the most prominent structures in these data, albeit with reduced significance.

The IC22 and IC40 data can be used to verify that the structures observed in the arrival direction distribution do not depend on the geometry of the detector or the data taking period. The shapes of both detector configurations are highly asymmetric, with a long axis and a short axis. The asymmetry introduces a trigger bias into the data, because muon tracks aligned with the long axis are much more likely to satisfy the simple majority trigger conditions than events arriving along the short axis. As a result, the local arrival direction distribution of the IC22 and IC40 data is highly nonuniform in azimuth.

We repeat the main analysis steps described in Sec.3, Fig.14]shows the angular power spectrum for IC22, IC40, and IC59. Both small- and large-scale structures are present in all three data sets.

Fig.15] shows the result of the dipole and quadrupole fits (left) and the residual map after 

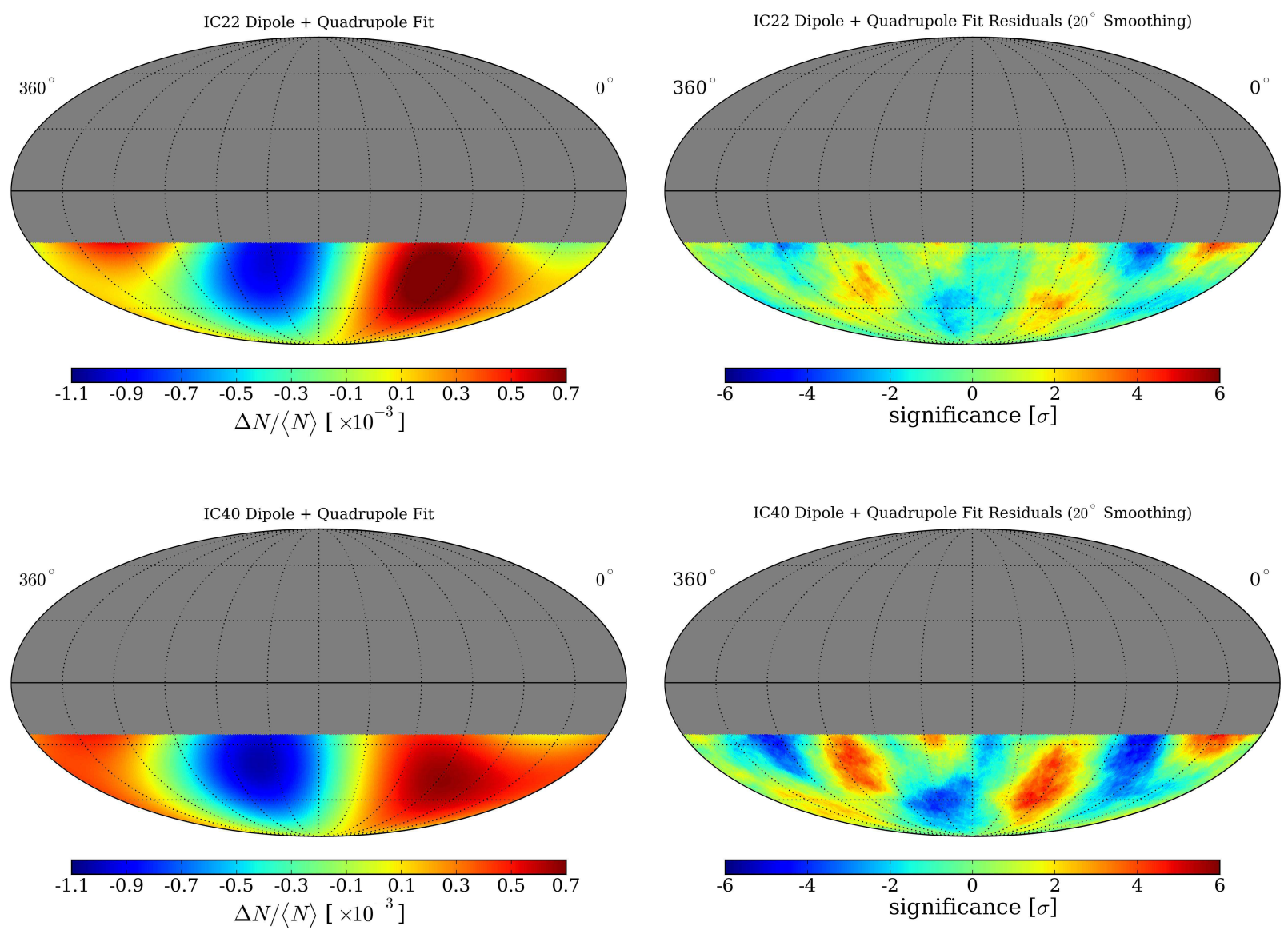

Fig. 15.- Top: Combined dipole and quadrupole fit of eq. (8) to data from IC22 (left) and fit residuals after $20^{\circ}$ smoothing (right). Bottom: Dipole and quadrupole fit to data from IC40 (left) and fit residuals (right).

subtraction of dipole and quadrupole (right) for IC22 (top) and IC40 (bottom). The residual maps are smoothed with a $20^{\circ}$ radius so they can be directly compared to Fig. 7 . While none of the features in IC22 and IC40 have a pre-trials significance above $5 \sigma$, they align with the regions of deficit and excess observed with IC59 data (cf. Fig. 7). The main features on both small and large scales appear to be persistent in all data sets.

Fig. 16 compares the results of the analysis described in Sec.3.5 for the IC22 and IC40 data. The figure shows the relative intensity as a function of right ascension for the declination band between $-45^{\circ}$ and $-30^{\circ}$, where the most significant deviations from isotropy are found. The systematic error band is estimated from the relative intensity distribution in anti-sidereal time as described in Sec.4.2, The results for IC22 (left) and IC40 (right) show that similar deviations are 

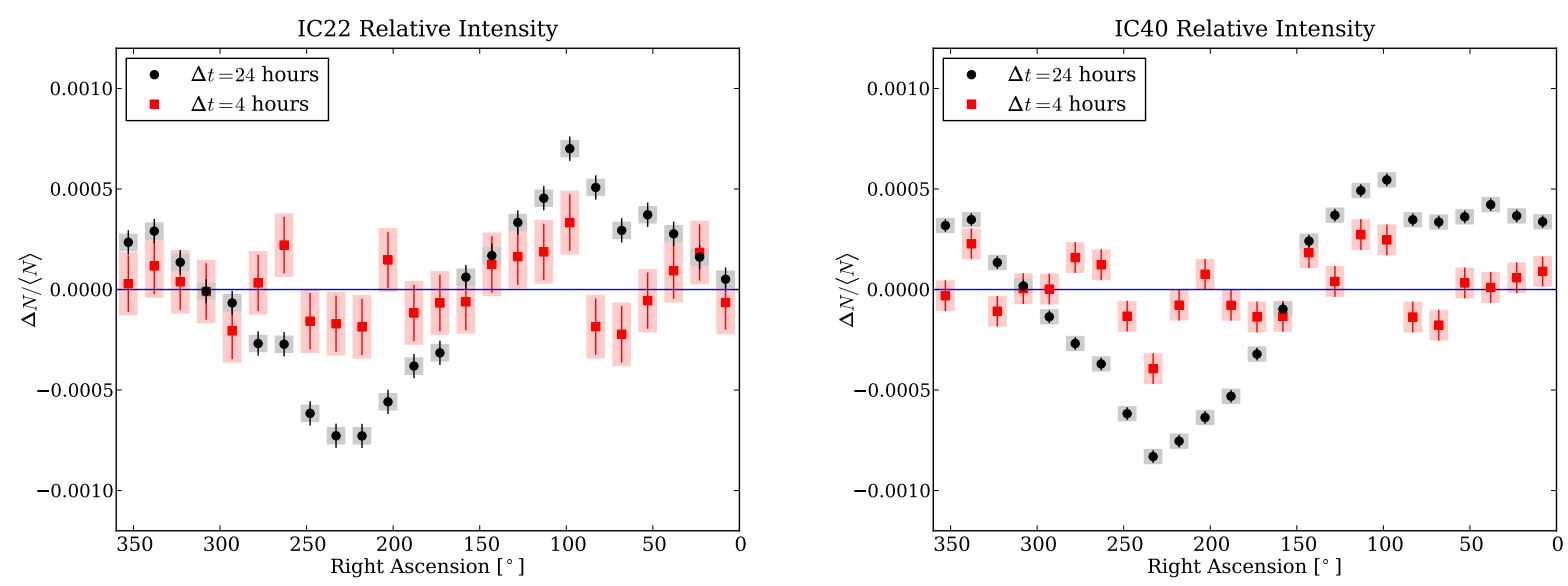

Fig. 16. - Relative intensity in the declination band between $-45^{\circ}$ and $-30^{\circ}$ for $\Delta t=4$ hours for data from IC22 (left) and IC40 (right). Statistical and systematic uncertainties are shown, with systematics calculated from the relative intensity distribution in anti-sidereal coordinates.

present in the IC22, IC40, and IC59 data, again with increasing significance due to the increasing size of the data sets.

The stability of the results over several years of data taking and three different detector configurations indicates that the anisotropy is not produced by the geometry of the detector. Since the temporal distribution of detector livetime is also different for all three data sets, the stability of the results indicates that the anisotropy is not affected by nonuniformities in the detector livetime. As expected, the time scrambling method accounts for this effect.

\section{Conclusions}

Using 32 billion events recorded with the partially-deployed IceCube detector between May 2009 and May 2010, we have shown that the arrival direction distribution of cosmic rays with a median energy of $20 \mathrm{TeV}$ exhibits significant anisotropy on all scales up to $\ell=12$ in the angular power spectrum. The power spectrum is dominated by a dipole and quadrupole moment, but also indicates the presence of significant structure on angular scales down to about $15^{\circ}$. These structures become visible in the skymap when the dominant dipole and quadrupole moments are either subtracted or suppressed. The residual skymap shows both significant excesses and deficits, with the most important excess reaching a post-trial significance of $5.3 \sigma$ in IC59. The relative intensity of the smaller-scale structures are about a factor of 5 weaker than the dipole and quadrupole structure. A study of data taken with the smaller IC22 and IC40 detectors in previous years confirms that these deviations from an isotropic flux are consistently present in all data sets. 


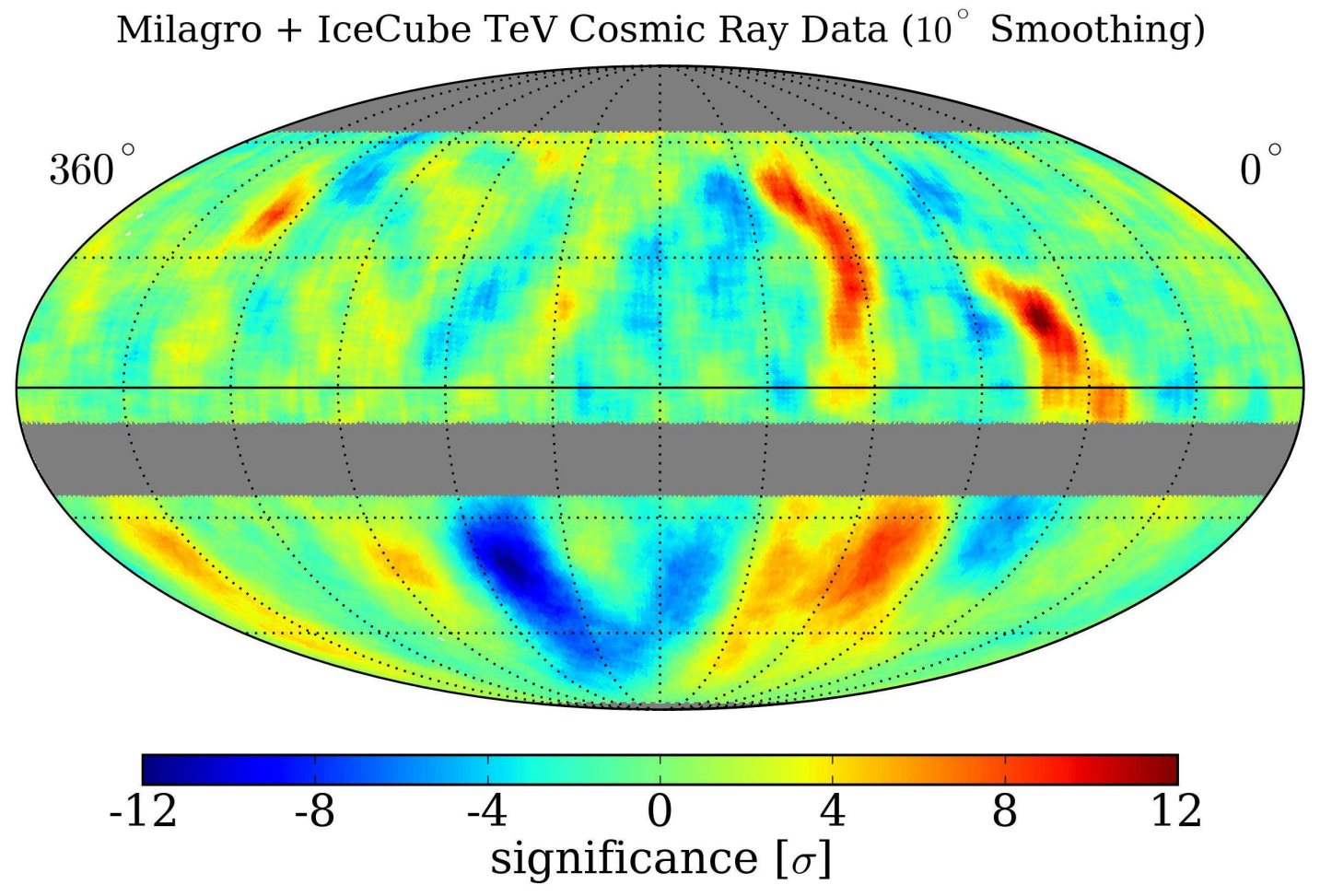

Fig. 17.- Combined map of significances in the cosmic ray arrival direction distribution observed by Milagro in the northern hemisphere (Abdo et al. 2008) and IceCube in the southern hemisphere (this analysis). Both maps have been smoothed with a $10^{\circ}$ radius.

Together with data from the $\gamma$-ray experiments in the northern hemisphere, we now have an almost complete cosmic ray map of the entire sky at TeV energies. Fig.17 shows the combined IceCube and Milagro skymaps of small-scale anisotropy. For this map, all available IceCube data (IC22, IC40, and IC59) have been used, with a total of $5.6 \times 10^{10}$ events, and the analysis is performed using the method described in Sec.3.5 with a smoothing radius of $10^{\circ}$ to match the Milagro analysis. The combined skymap shows significant excess regions in both hemispheres. It is possible that the structure around right ascension $120^{\circ}$ spans both hemispheres, as the drop in significances around declination $\delta=0^{\circ}$ could be an artifact of the smaller exposure of both detectors near $\delta=0^{\circ}$, which corresponds to a region close to the horizon for both detectors.

There is currently no explanation for these local enhancements in the cosmic ray flux. We note that the two most significant excess regions in the southern sky (regions 1 and 2 in Tab. 2) are both located near the Galactic plane. In addition, the position of one of the excess regions (region 1) coincides with the location of the Vela pulsar at $\left(\alpha=128.8^{\circ}, \delta=-45.2^{\circ}\right)$. At a distance of about 300 pc (Caraveo et al. 2001), Vela is one of the closest known supernova remnants, and 
has long been considered a candidate source for Galactic cosmic ray acceleration. However, the Larmor radius of $10 \mathrm{TeV}$ protons in a $\mu \mathrm{G}$ magnetic field is approximately $0.01 \mathrm{pc}$, many orders of magnitude smaller than the distance to Vela, and unless unconventional propagation mechanisms are assumed, charged particles from Vela will have lost all directional information upon their arrival at Earth.

Recently, several authors have investigated the extent to which the stochastic nature of nearby supernova remnants can lead to spatial and temporal variations in the cosmic ray flux (Ptuskin et al. 2006; Blasi \& Amato 2011). The random nature of the sources makes quantitative predictions difficult, and can lead to bumps and dips in the amplitude of the anisotropy as a function of energy that depend on the specific source distribution used in the simulation of the cosmic ray flux. Qualitatively, the models make specific predictions for the energy dependence of the amplitude of the cosmic ray anisotropy.

In the $\mathrm{TeV}-\mathrm{PeV}$ range, the energy resolution of IceCube is poor for cosmic ray events (see Sec. 2.1). However, given the large rate of cosmic ray triggers, it is possible to isolate a sufficiently large subset of showers with a median energy of several hundred $\mathrm{TeV}$ which is not significantly contaminated by low energy events. A paper focusing on this study is currently in preparation.

The study of cosmic ray arrival directions at $\mathrm{TeV}$ energies will continue to be a major ongoing research effort in IceCube. IceCube and the future High Altitude Water Cherenkov (HAWC) $\gamma$-ray observatory (Sinnis et al. 2004) under construction in Mexico can be used to monitor the southern and northern hemisphere, respectively, with high sensitivity. The combined data sets will soon allow for all-sky power spectra and the analysis of the entire sky at all angular scales.

Over the next few years, with the IceCube detector now operating in its complete 86-string configuration, our data set will increase at a rate of about $45 \times 10^{9}$ muon events per year. With this level of statistics we will also be able to study possible time dependencies of the anisotropy in the southern hemisphere and compare to similar studies performed with data from instruments in the northern hemisphere (Abdo et al. 2009; Amenomori et al. 2010).

\section{Acknowledgments}

We would like to thank Eric Hivon for helpful comments and suggestions about the angular power spectrum analysis, and the Milagro Collaboration for providing us with their data to produce the combined skymap in Fig.17.

Some of the results in this paper have been derived using the HEALPix (Gorski et al. 2005) and the PolSpice (Szapudi et al. 2001; Chon et al. 2004) software libraries.

We acknowledge the support from the following agencies: U.S. National Science FoundationOffice of Polar Programs, U.S. National Science Foundation-Physics Division, University of Wisconsin Alumni Research Foundation, the Grid Laboratory Of Wisconsin (GLOW) grid infrastructure 
at the University of Wisconsin - Madison, the Open Science Grid (OSG) grid infrastructure; U.S. Department of Energy, and National Energy Research Scientific Computing Center, the Louisiana Optical Network Initiative (LONI) grid computing resources; National Science and Engineering Research Council of Canada; Swedish Research Council, Swedish Polar Research Secretariat, Swedish National Infrastructure for Computing (SNIC), and Knut and Alice Wallenberg Foundation, Sweden; German Ministry for Education and Research (BMBF), Deutsche Forschungsgemeinschaft (DFG), Research Department of Plasmas with Complex Interactions (Bochum), Germany; Fund for Scientific Research (FNRS-FWO), FWO Odysseus programme, Flanders Institute to encourage scientific and technological research in industry (IWT), Belgian Federal Science Policy Office (Belspo); University of Oxford, United Kingdom; Marsden Fund, New Zealand; Japan Society for Promotion of Science (JSPS); the Swiss National Science Foundation (SNSF), Switzerland; A. Groß acknowledges support by the EU Marie Curie OIF Program; J. P. Rodrigues acknowledges support by the Capes Foundation, Ministry of Education of Brazil.

\section{REFERENCES}

Abbasi, R., et al. 2009a, Astrophys. J., 701, L47

—. 2009b, Nucl. Instrum. Meth., A601, 294

—. 2010a, Nucl. Instrum. Meth., A618, 139

—. 2010b, Astrophys. J., 718, L194

Abdo, A. A., et al. 2008, Phys. Rev. Lett., 101, 221101

—. 2009, Astrophys. J., 698, 2121

Achterberg, A., et al. 2006, Astropart. Phys., 26, 155

Aglietta, M., et al. 2009, Astrophys. J. Lett., 692, L130

Ahn, E.-J., et al. 2009, Phys. Rev., D80, 094003

Ahrens, J., et al. 2004, Nucl. Instrum. Meth., A524, 169

Alexandreas, D. E., et al. 1993, Nucl. Instrum. Meth., A328, 570

Amenomori, M., et al. 2004, Phys. Rev. Lett., 93, 061101

—. 2005, Astrophys. J., 626, L29

—. 2006, Science, 314, 439

—. 2008, Astrophys. J., 672, L53 
—. 2010, Astrophys. J., 711, 119

Ansari, R., \& Magneville, C. 2010, Mon. Not. Roy. Astron. Soc., 405, 1421

Biermann, P. L., et al. 2010, Astrophys. J., 725, 184

Blasi, P., \& Amato, E. 2011, submitted to JCAP, arXiv:1105.4529v1

Caraveo, P., et al. 2001, Astrophys. J., 561, 930

Chon, G., et al. 2004, Mon. Not. Roy. Astron. Soc., 350, 914

Compton, A. H., \& Getting, I. A. 1935, Phys. Rev., 47, 817

Drury, L., \& Aharonian, F. 2008, Astropart. Phys., 29, 420

Gleeson, L., \& Axford, W. 1968, Astrophysics and Space Science, 2, 431, 10.1007/BF02175919

Gorski, K. M., et al. 2005, Astrophys. J., 622, 759

Guillian, G., et al. 2007, Phys. Rev. D, 75, 062003

Heck, D., et al. 1998, Forschungszentrum Karlsruhe Report FZKA 6019

Hörandel, J. R. 2003, Astropart. Phys., 19, 193

Lazarian, A., \& Desiati, P. 2010, Astrophys. J., 722, 188

Li, T.-P., \& Ma, Y.-Q. 1983, Astrophys. J., 272, 317

Malkov, M. A., et al. 2010, Astrophys. J., 721, 750

Ptuskin, V., Jones, F., Seo, E., \& Sina, R. 2006, Advances in Space Research, 37, 1909

Sinnis, G., Smith, A., \& McEnery, J. E. 2004, in Tenth Marcel Grossman Meeting, Rio de Janeiro, Brazil, 1068-1088

Smoot, G. F., \& Lubin, P. M. 1979, Astrophys. J., 234, L83

Szapudi, I., Prunet, S., Pogosyan, D., Szalay, A. S., \& Bond, J. R. 2001, Astrophys. J., 548, L115

Tilav, S., et al. 2009, in Proc. 31st ICRC, Łódź, Poland

Vernetto, S., et al. 2009, in Proc. 31st ICRC, Łódź, Poland 